\title{
Berry's phases of ground states of interacting spin-one bosons: Chains of monopoles and monosegments
}

\author{
Jeroen Wiemer ${ }^{1}$ and Fei Zhou ${ }^{1,2, *}$ \\ ${ }^{1}$ ITP, Utrecht University, Leuvenlaan 4, 3584 CE Utrecht, The Netherlands \\ ${ }^{2}$ Department of Physics and Astronomy, University of British Columbia, 6224 Agriculture Road, Vancouver, BC, Canada V6T 1Z1
}

(Received 5 November 2003; published 27 September 2004)

\begin{abstract}
We study Berry's connection potentials of many-body ground states of spin-one bosons with antiferromagnetic interactions in adiabatically varying magnetic fields. We find that Berry's connection potentials are generally determined by, instead of usual singular monopoles, linearly positioned monosegments each of which carries one unit of topological charge; in the absence of a magnetic field gradient this distribution of monosegments becomes a linear chain of monopoles. Consequently, Berry's phases consist of a series of step functions of magnetic fields; a magnetic field gradient causes rounding of these step functions. We also calculate Berry's connection fields, profiles of monosegments, and show that the total topological charge is conserved in a parameter space.
\end{abstract}

DOI: $10.1103 /$ PhysRevB.70.115110

PACS number(s): 03.75.Mn, 05.30.Jp, 75.10.Jm

\section{INTRODUCTION}

In the presence of time-dependent periodical potentials $V(t)=V(t+T)$ with a period $T$, an eigenstate of the instantaneous Hamiltonian acquires a geometric phase known as the Berry's phase. ${ }^{1}$ The Berry's phase is a global characterization of an eigenvector when it is transported in a Hilbert space. For a period of $T$ during which an eigenstate $\Psi[V(t)]$ evolves adiabatically, the Berry's phase is simply $\Phi_{B}=-\operatorname{Im} \int_{0}^{T}\left\langle\Psi\left|\partial_{t}\right| \Psi\right\rangle d t$. From the point of view of fiber bundles, the Berry's phase can be considered as the holonomy of a Hermitian fiber bundle; its base space corresponds to a parameter space and a fiber is an eigenstate which defines a mapping from the parameter space to the Hilbert space. This point of view was illustrated in Ref. 2. Berry's phases have been observed in a variety of experiments such as nuclear magnetic resonance (NMR) and rotation of light polarization in optical fibers, etc. ${ }^{3-6}$

In condensed matter systems where there are large numbers of particles interacting with each other one way or the other, the subject of Berry's phases, or more general geometric phases becomes more fascinating and intriguing. There are at least two interesting aspects of this subject: (i) the effect of geometric phases on correlations and (ii) the effect of correlations on Berry's phases.

The first issue was addressed on quite a few different occasions. The best known result perhaps is the geometric phases' effect on spin correlations. As pointed out a while ago for antiferromagnets, a Berry's phase distinguishes integer and half-integer spin chains, or even-integer, odd-integer and half-integer spin square lattices and results in different ground states and excitations. ${ }^{7-9}$ The other examples perhaps are the possible geometric phases' effects on statistical transmutation and fractionalization in spin correlated systems. ${ }^{10-13}$ In mesoscopic magnetic systems effects of Berry's phases on collective quantum tunneling were investigated; ${ }^{14-19}$ quantum interferences of Berry's phases were observed in molecular magnets. ${ }^{20}$

In this paper we are going to address the second aspect, correlations' effect on Berry's phases. We study Berry's con- nection potentials (one-form), Berry's connection fields (two-form), and topological charge densities for ground states of spin-one bosons with antiferromagnetic interactions. In a parameter space

$$
\left\{X_{a}\right\}, \quad a=1,2, \ldots, M,
$$

the connection potential $\mathbf{A}_{a}$, two-form connection fields $\mathbf{F}_{a b}$, and topological charge and current densities $(\rho, \mathbf{J})$ are defined as

$$
\begin{gathered}
\mathbf{A}_{a}\left(\left\{X_{c}\right\} ; \Psi\right)=\frac{i}{2}\left\{\left\langle\Psi \mid \frac{\partial \Psi}{\partial X_{a}}\right\rangle-\left\langle\frac{\partial \Psi}{\partial X_{a}} \mid \Psi\right\rangle\right\}, \\
\mathbf{F}_{a b}\left(\left\{X_{c}\right\} ; \Psi\right)=i\left\{\left\langle\frac{\partial \Psi}{\partial X_{a}} \mid \frac{\partial \Psi}{\partial X_{b}}\right\rangle-\left\langle\frac{\partial \Psi}{\partial X_{b}} \mid \frac{\partial \Psi}{\partial X_{a}}\right\rangle\right\}, \\
4 \pi \rho=\frac{1}{2} \epsilon^{a b c} \partial_{a} \mathbf{F}_{b c}, \quad 4 \pi \mathbf{J}_{a}=\partial_{b} \mathbf{F}_{a b} .
\end{gathered}
$$

Consider Berry's connection potentials, two-form connection fields and topological charges when $N$ spin-one bosons are in a magnetic field $\mathbf{B}=\left(B_{x}, B_{y}, B_{z}\right)$. For ground states of $N$-noninteracting spin-one bosons, one easily confirms that the two-form connection fields are monopole-like due to an $N$-fold magnetic monopole at the point of $\mathbf{B}=0$ in the parameter space of $\left\{B_{a}\right\}, a=1,2,3$ or $x, y, z$. And the topological current is zero. That is

$$
\begin{gathered}
\mathbf{b}_{a}=\frac{1}{2} \epsilon_{a b c} F_{b c}=Q(\mathbf{B}) \frac{\mathbf{B}_{a}}{|\mathbf{B}|^{3}}, \quad Q(\mathbf{B})=N, \\
\rho=Q(\mathbf{B}) \delta(\mathbf{B}), \quad \mathbf{J}=0 .
\end{gathered}
$$

We should emphasize here that the topological charge is localized at the origin $\mathbf{B}=0$ in the parameter space. Note that the Berry's two-form field $\mathbf{b}$ is a function of external magnetic fields $\mathbf{B}$.

For $N$ spin-one bosons with antiferromagnetic interactions, we are going to show that the profile of topological charge density is a linear chain of monopoles. In the pres- 
ence of a field gradient, we also find nonsingular monosegments where topological charges distribute smoothly along certain direction and topological current flows. And the density profile is a linear chain of monosegments. Details of topological charge density profile depend on the even-odd parity of $N$, magnetic field gradient and spin relaxation.

The plan of this paper is as follows. In Sec. II, we introduce the system which interests us and describe the algebras needed for this investigation. In Sec. III, we calculate the Berry's connection fields of $N$ interacting spin-one bosons in a uniform magnetic field. We show that antiferromagnetic interactions in general expel the topological charges outward from the origin and result in a linear chain distribution of monopoles in the parameter space. In Sec. IV, we study the effects of the spin nonconserving process; we calculate the monosegment profile in the presence of a magnetic field gradient. In addition, we address the Landau-Zener effect.

In both Secs. III and IV, we show that Berry's phases are suppressed because of antiferromagnetic interactions and as functions of magnetic field consist of a series of step-like functions. Furthermore, the shape of steps is determined by a magnetic field gradient. We note that in the absence of interactions, the many-body Berry's phase is simply the sum of each individual spin-one bosons and does not depend on magnetic fields.

In Sec. IV we outline an alternative description of interacting spin-one bosons using quantum rotor models. In the effective representation, we show that the problem of interacting spin-one bosons in a magnetic field gradient is equivalent to a quantum rotor coupled to a quadrupole field.

\section{THE MICROSCOPIC HAMILTONIAN}

We consider spin-one bosons in an optical trap in the dilute limit defined by $n a^{3} \ll 1$ where $a$ is the $s$-wave scattering length and $n$ the density. The Hamiltonian is given as

$$
\begin{aligned}
H= & \int d \mathbf{r}\left\{\frac{\hbar^{2}}{2 m} \boldsymbol{\nabla} \hat{\psi}_{\alpha}^{\dagger}(\mathbf{r}) \cdot \boldsymbol{\nabla} \hat{\psi}_{\alpha}(\mathbf{r})+U(\mathbf{r}) \hat{\psi}_{\alpha}^{\dagger}(\mathbf{r}) \hat{\psi}_{\alpha}(\mathbf{r})\right. \\
& +\gamma \mathbf{B} \cdot \hat{\psi}_{\alpha}^{\dagger}(\mathbf{r}) \mathbf{S}_{\alpha \beta} \hat{\psi}_{\beta}(\mathbf{r})+\frac{c_{0}}{2} \hat{\psi}_{\alpha}^{\dagger}(\mathbf{r}) \hat{\psi}_{\alpha^{\prime}}^{\dagger}(\mathbf{r}) \hat{\psi}_{\alpha^{\prime}}(\mathbf{r}) \hat{\psi}_{\alpha}(\mathbf{r}) \\
& \left.+\frac{c_{2}}{2} \hat{\psi}_{\alpha}^{\dagger}(\mathbf{r}) \hat{\psi}_{\alpha^{\prime}}^{\dagger}(\mathbf{r}) \mathbf{S}_{\alpha \beta} \cdot \mathbf{S}_{\alpha^{\prime} \beta^{\prime}} \hat{\psi}_{\beta^{\prime}}(\mathbf{r}) \hat{\psi}_{\beta}(\mathbf{r})\right\}
\end{aligned}
$$

Interactions between atoms are approximated by spindependent contact interactions.

In a single mode approximation, the creation and annihilation operators are defined as

$$
\hat{\psi}_{\alpha}^{\dagger}(\mathbf{r})=\hat{\psi}_{\alpha}^{\dagger} \chi_{0}^{*}(\mathbf{r})
$$

$\alpha=m=0, \pm 1 . \chi_{0}(\mathbf{r})$ is the lowest orbital mode and $|1, m\rangle$ is a spin-one state with $S_{z}=m ; \mathbf{S}_{\alpha \beta}$ are three matrices

$$
S^{x}=\frac{1}{\sqrt{2}}\left(\begin{array}{ccc}
0 & 1 & 0 \\
1 & 0 & 1 \\
0 & 1 & 0
\end{array}\right), \quad S^{y}=\frac{1}{\sqrt{2}}\left(\begin{array}{ccc}
0 & -i & 0 \\
i & 0 & -i \\
0 & i & 0
\end{array}\right),
$$

$$
S^{z}=\left(\begin{array}{ccc}
1 & 0 & 0 \\
0 & 0 & 0 \\
0 & 0 & -1
\end{array}\right) .
$$

The dilute limit of spin-one bosons with antiferromagnetic interactions was studied in a few experiments in Refs. 21 and 22 and also theoretically investigated in various works. ${ }^{23-26}$ A geometric-description-based nonperturbative approach to strong-coupling limits was proposed in Refs. 27 and 28. In optical lattices, correlated Mott states of spin-one bosons were studied in a series of recent papers $;{ }^{29-32}$ spin nematic, spin singlet and dimerized-valence-bond crystals were found for high dimensional and low dimensional optical lattices. Unconventional spin disordered condensates in homogeneous limits were proposed in various papers, ${ }^{28,33}$ many properties of spin singlet condensates were further explored in low dimensional optical lattices. ${ }^{34}$ In fast rotating traps, correlated quantum liquids of spin-one bosons were also investigated. ${ }^{35,36}$

It is convenient to introduce the following creationannihilation operators:

$$
\begin{gathered}
\hat{\psi}_{x}^{\dagger}=\frac{1}{\sqrt{2}}\left(\hat{\psi}_{-1}^{\dagger}-\hat{\psi}_{1}^{\dagger}\right), \\
\hat{\psi}_{y}^{\dagger}=\frac{i}{\sqrt{2}}\left(\hat{\psi}_{-1}^{\dagger}+\hat{\psi}_{1}^{\dagger}\right), \\
\hat{\psi}_{z}^{\dagger}=\hat{\psi}_{0}^{\dagger} .
\end{gathered}
$$

In this representation total spin operators are defined as

$$
\hat{\mathbf{S}}^{\alpha}=\hat{\psi}_{\beta}^{\dagger} \mathbf{S}_{\beta \gamma}^{\alpha} \hat{\psi}_{\gamma}, \quad \mathbf{S}_{\beta \gamma}^{\alpha}=-i \epsilon_{\alpha \beta \gamma}
$$

$\hat{\psi}_{\alpha}^{\dagger}, \hat{\psi}_{\alpha}$ (from now on, $\alpha=x, y, z$ ) are usual bosonic operators obeying the following commutation relations:

$$
\left[\hat{\psi}_{\alpha}, \hat{\psi}_{\beta}\right]=\left[\hat{\psi}_{\alpha}^{\dagger}, \hat{\psi}_{\beta}^{\dagger}\right]=0,\left[\hat{\psi}_{\alpha}, \hat{\psi}_{\beta}^{\dagger}\right]=\delta_{\alpha \beta} .
$$

Using these results, the Hamiltonian can be written in the following form:

$$
H=\left(\epsilon_{0}-g_{0}-2 g_{2}\right) \hat{N}+g_{0} \hat{N}^{2}+g_{2} \hat{\mathbf{S}}_{\text {tot }}^{2}+\gamma \mathbf{B} \cdot \hat{\mathbf{S}}_{\text {tot }}
$$

with

$$
\begin{aligned}
& g_{0}=\frac{c_{0}}{2} \int d \mathbf{r}\left|\chi_{0}(\mathbf{r})\right|^{4} \\
& g_{2}=\frac{c_{2}}{2} \int d \mathbf{r}\left|\chi_{0}(\mathbf{r})\right|^{4} .
\end{aligned}
$$

In terms of singlet pair creation operators $\hat{A}$ :

$$
\hat{A}^{\dagger}|0\rangle=\frac{1}{\sqrt{6}}\left(-2 \hat{\psi}_{-1}^{\dagger} \hat{\psi}_{1}^{\dagger}+\hat{\psi}_{0}^{\dagger} \hat{\psi}_{0}^{\dagger}\right)|0\rangle,
$$

the total spin operator can also be written as 


$$
\hat{\mathbf{S}}_{\text {tot }}^{2}=\hat{N}(\hat{N}+1)-6 \hat{A}^{\dagger} \hat{A},
$$

with $\hat{N}=\hat{\psi}_{\alpha}^{\dagger} \hat{\psi}_{\alpha}$ the number operator. Consequently, we rewrite the Hamiltonian as

$$
H=\left(\epsilon_{0}-g_{0}-g_{2}\right) \hat{N}+\left(g_{0}+g_{2}\right) \hat{N}^{2}-6 g_{2}\left(\hat{A}^{\dagger} \hat{A}\right)+\gamma \mathbf{B} \cdot \hat{\mathbf{S}}_{\mathrm{tot}} .
$$

\section{A. Spectrum}

The Hamiltonian commutes with the number operator, square of the total spin operator $\hat{\mathbf{S}}_{\text {tot }}^{2}$, and operator $\hat{A}^{\dagger} \hat{A}$. Eigenstates of the Hamiltonian are simultaneous eigenstates of the number operator $\hat{N}$ and operator $\hat{A}^{\dagger} \hat{A}$. If $\left|N, N_{s}\right\rangle$ is a state with a total number of $N$ particles and $N_{s}$ pairs of singlets, then we have

$$
\hat{\mathbf{S}}_{\text {tot }}^{2}\left|N, N_{s}\right\rangle=\left(N-2 N_{s}\right)\left(N-2 N_{s}+1\right)\left|N, N_{s}\right\rangle
$$

or $S=N-2 N_{s}$.

For $N$ particles in a magnetic field $\mathbf{B}$ along the $z$ axis, the spin Hamiltonian is

$$
H=g_{2} \hat{\mathbf{S}}_{\mathrm{tot}}^{2}+\gamma \mathbf{B} \cdot \hat{\mathbf{S}}_{\mathrm{tot}} .
$$

Obviously, an external magnetic field along the $z$ axis splits the $2 S+1$-fold degeneracy of states with given spin $S$ and $\mathbf{S}^{z}$ remains to be a good quantum number. The energy of an eigen state $\left|S, S_{z}=m\right\rangle$ is

$$
E_{S, m}=g_{2} S(S+1)+\gamma B m
$$

The lowest energy state for a given spin $S$ is $|S,-S\rangle$. The low energy spectrum at different magnetic fields can be found in Fig. 1.

\section{B. Level crossings}

\section{1. $N$ odd}

Levels $\left|S_{1}, m_{1}\right\rangle$ and $\left|S_{2}, m_{2}\right\rangle$ cross at

$$
B=\frac{g_{2}}{\gamma} \frac{S_{2}\left(S_{2}+1\right)-S_{1}\left(S_{1}+1\right)}{m_{1}-m_{2}} .
$$

For the following values of magnetic fields

$$
B_{k}=\frac{g_{2}}{\gamma}(4 k+5)
$$

with $k=0,1,2, \ldots, M_{N}$ where $M_{N}=(N-3) / 2$ for odd $N$, we have level crossings in ground states.

\section{2. $N$ even}

For an even $N$, the level crossings in the ground state take place at

$$
B_{k}=\frac{g_{2}}{\gamma}(4 k+3),
$$

with $k=0,1,2, \ldots, M_{N}$ where $M_{N}=(N-2) / 2$ for even $N$.
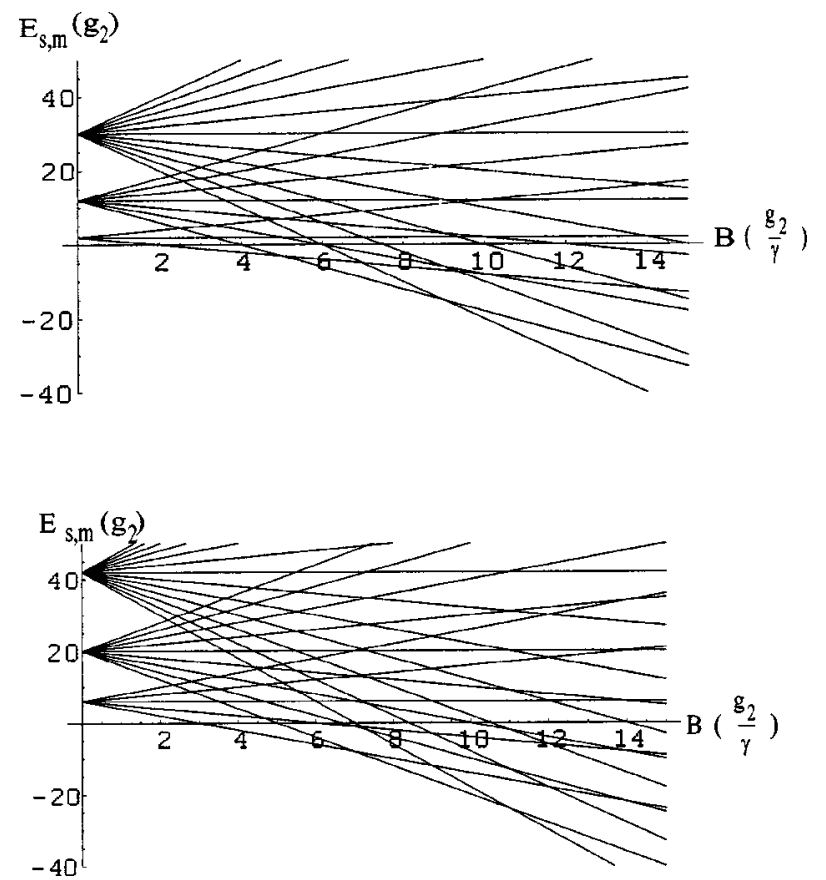

FIG. 1. Energy spectrum as a function of magnetic field. $E_{s, m}$ is given in units of $g_{2}, B$ in units of $g_{2} \gamma^{-1}$. (a) is for an odd $N$ and (b) is for an even $N$.

\section{MONOPOLES AND BERRY'S CONNECTION FIELDS OF $N$ INTERACTING SPIN-ONE BOSONS IN HOMOGENEOUS MAGNETIC FIELDS}

\section{A. Local connection fields and topological charge densities}

The microscopic many-body state of $\left|S, S_{z}=-S\right\rangle$ is given as

$$
\left|S, S_{z}=-S\right\rangle=C\left(\hat{\psi}_{-1}^{\dagger}\right)^{S} \hat{A}^{(N-S) / 2}|0\rangle .
$$

Here $\hat{A}$ is the singlet creation operator defined before; $C$ is a normalization factor

$$
C=6^{(N-S) / 4} \frac{1}{\sqrt{f\left(\frac{N-S}{2}, S\right)}}
$$

with

$$
f(M, S)=S ! M ! 2^{M} \frac{(2 M+2 S+1) ! !}{(2 S+1) ! !} .
$$

In a spherical coordinate system, we denote a magnetic field $\mathbf{B}$, of magnitude $B$ pointing in the direction of $\mathbf{n}$ $=(\cos \phi \sin \theta, \sin \phi \sin \theta, \cos \theta)$ as

$$
\mathbf{B}=(B, \theta, \phi) .
$$

For a magnetic field $\mathbf{B}$ pointing at $\mathbf{n}$ direction, ground states

$$
|g\rangle=\Psi_{S(B),-S(B)}(\mathbf{n})
$$

are states $\left|S, S_{z}=-S ; \mathbf{n}\right\rangle$ with the $z$ axis defined along the $\mathbf{n}$ direction. The corresponding many-body wave functions are 


$$
\begin{aligned}
\Psi_{S(B),-S(B)}(\mathbf{n})= & C\left[-i \sin ^{2}\left(\frac{\theta}{2}\right) e^{-i \phi} \hat{\psi}_{+1}^{\dagger}+\frac{i}{\sqrt{2}} \sin \theta \hat{\psi}_{0}^{\dagger}\right. \\
& \left.-i \cos ^{2}\left(\frac{\theta}{2}\right) e^{i \phi} \hat{\psi}_{-1}^{\dagger}\right]^{S} \hat{A}^{\dagger(N-S) / 2}|0\rangle .
\end{aligned}
$$

And finally $S$ is a function of $B$, the magnitude of external magnetic fields. As shown in Fig. 1:

$$
S=S_{0}(N)+2 \sum_{k=0,1,2 \ldots}^{M_{N}} \Theta\left(|\mathbf{B}|-B_{k}\right) .
$$

$S_{0}(N)$ is zero for an even $N$ but is unity for an odd $N$.

For these correlated ground states, Berry's connection potentials in spherical coordinates $(B, \theta, \phi)$ can be defined as

$$
\begin{aligned}
\mathbf{A}(\mathbf{B})= & -\operatorname{Im}\left\langle\Psi_{S,-S}(\mathbf{n})\left|\frac{\partial}{\partial B}\right| \Psi_{S,-S}(\mathbf{n})\right\rangle \mathbf{e}_{B} \\
& -\frac{1}{B} \operatorname{Im}\left\langle\Psi_{S,-S}(\mathbf{n})\left|\frac{\partial}{\partial \theta}\right| \Psi_{S,-S}(\mathbf{n})\right\rangle \mathbf{e}_{\theta} \\
& -\frac{1}{B \sin \theta} \operatorname{Im}\left\langle\Psi_{S,-S}(\mathbf{n})\left|\frac{\partial}{\partial \phi}\right| \Psi_{S,-S}(\mathbf{n})\right\rangle \mathbf{e}_{\phi} .
\end{aligned}
$$

A direct calculation of $\mathbf{A}$ yields desired results

$$
\begin{gathered}
\mathbf{A}=-\frac{\cos \theta}{\sin \theta} \frac{Q(|\mathbf{B}|)}{|\mathbf{B}|} \mathbf{e}_{\phi} ; \\
Q(|\mathbf{B}|)=q_{0}(N)+2 \sum_{k=0,1,2 \ldots}^{M_{N}} \Theta\left(|\mathbf{B}|-B_{k}\right) .
\end{gathered}
$$

$q_{0}(N)=1$ for an odd number of particles and $q_{0}(N)=0$ for an even number of particles. The two-form connection fields are

$$
\mathbf{b}=Q(|\mathbf{B}|) \frac{1}{|\mathbf{B}|^{2}} \mathbf{e}_{B}+\frac{1}{B} \frac{\partial Q(|\mathbf{B}|)}{\partial B} \frac{\cos \theta}{\sin \theta} \mathbf{e}_{\theta} .
$$

It is worth emphasizing that the radial component of $\mathbf{b}$ fields can be attributed to multiple shells, each of which carries exactly two units of charges and is located at $B=B_{k}$. Indeed, if one defines $4 \pi \rho_{B}=\nabla \cdot\left(\mathbf{b} \cdot \mathbf{e}_{B}\right) \mathbf{e}_{B}$, one obtains

$$
\rho_{B}=q_{0}(N) \delta(\mathbf{B})+\frac{1}{2 \pi} \sum_{k=0,1,2 \ldots}^{M_{N}} \delta\left(B-B_{k}\right) \frac{1}{B^{2}},
$$

which indicates an isotropic topological charge distribution of a series of shells at $B=B_{k}$.

The topological charge due to the $\theta$ component of $\mathbf{b}$ fields on the other hand consists of two contributions: (a) an isotropic charge distribution of spherical shells exactly identical to $-\rho_{B}$ given earlier; and (b) linearly distributed monopoles located at $\mathbf{B}= \pm B_{k} \mathbf{e}_{z}$ each of which also carries one unit charge. As a result, the total amount of topological charge due to the $\theta$ component vanishes identically on each shell; however, the $\theta$ component deforms the isotropic distribution on each shell completely.

Finally, the total topological charge and current densities are

$$
\begin{gathered}
\rho=q_{0} \delta(\mathbf{B})+\frac{1}{2 \pi \sin \theta|\mathbf{B}|^{2}} \sum_{k=0,1, \ldots}^{M_{N}} \delta\left(|\mathbf{B}|-B_{k}\right) \\
\times[\delta(\theta-\pi)+\delta(\theta)], \\
\mathbf{J}=\frac{1}{4 \pi|\mathbf{B}|} \frac{\partial^{2} Q(|\mathbf{B}|)}{\partial B^{2}} \cot (\theta) \mathbf{e}_{\phi} .
\end{gathered}
$$

Compared with noninteracting systems, we find the following three new features in Berry's connection fields and topological charges.

(i) Following Eqs. (29) and (31), we find that the total topological charge within the $k$ th shell bounded by an outer surface $B_{1}=B_{k}+h$ and an inner surface $B_{2}=B_{k}-h$, where $B_{k+1}-B_{k} \gg h$ is a conserved integer independent of $B$ and index $k$. Indeed

$$
\frac{1}{4 \pi} \oint_{S_{1}} B \cdot e_{B} d S-\frac{1}{4 \pi} \oint_{S_{2}} B \cdot e_{B} d S=2 .
$$

Here the integration is over two surfaces $S_{1,2}$ of $B=B_{1,2}$.

Therefore, each shell defined earlier still carries over all two units of topological charges. If we introduce the total charge of a surface with radius $B$ as

$$
\sigma(B)=2 \pi \int \rho(B, \theta) B^{2} \sin \theta d \theta,
$$

we find

$$
\sigma(B)=q_{0}(N) \delta(B)+2 \sum_{k=0,1, \ldots}^{M_{N}} \delta\left(|\mathbf{B}|-B_{k}\right) .
$$

At last, topological currents on each shell circulate around the $z$ axis.

As shown in the introduction, for noninteracting spin-one bosons, the topological charge is located at the center of the parameter space. Following Eqs. (31) and (34) it is evident that antiferromagnetic interactions between spin-one bosons lead to expulsion of topological charges from the origin $\mathbf{B}$ $=0$. As a result, topological charges distribute on different shells with radius $B_{k}, k=0,1, \ldots, M_{N}$; each shell carries two units of charges.

However, following Eq. (31), one also confirms that the total topological charge $Q_{T}$ is conserved; $Q_{T}$ is exactly the number of particles in the many-body state, independent of interaction strength. That is

$$
Q_{T}=\int \rho(\mathbf{B}) d \mathbf{B}=N .
$$

One can easily show that the solutions in Eqs. (34) and (35) are independent of the specific class of ground state wave functions chosen for this investigation, though the results in Eq. (31) do depend on choices of wave functions.

(ii) Furthermore, on each shell the distribution of $\mathbf{b}$ fields breaks the rotational symmetry, unlike in noninteracting cases; consequently the charge distribution on each shell is highly anisotropic. The expulsion of topological charges for the particular set of states studied here is along the $z$ axis only. One can verify that in the case of homogeneous mag- 

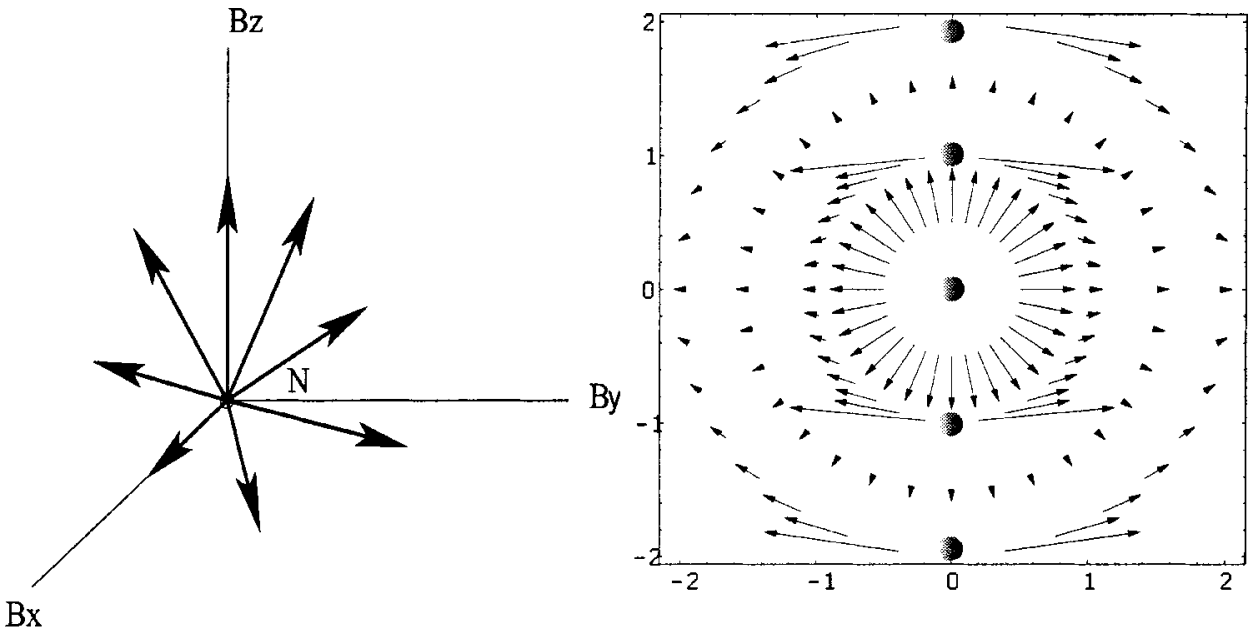

a)

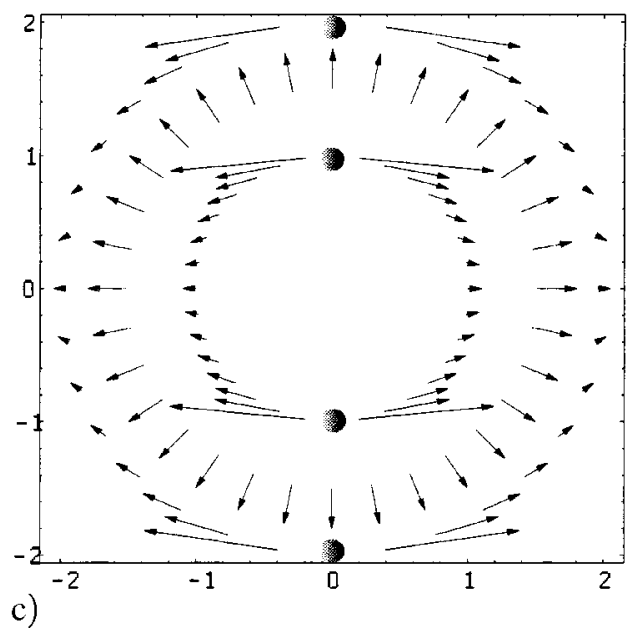

FIG. 2. Schematic of topological field and charge distribution in the parameter space $\left(B_{x}, B_{y}, B_{z}\right)$. In (a) we show the topological field and charge distribution for $\mathrm{N}$-noninteracting spin-one bosons. In (b) and (c) we show the field and charge distribution for an odd and even number of spin-one bosons with antiferromagnetic interactions respectively; chains of monopoles are shown here explicitly. The b fields only have radial component except on shells of $B$ $=B_{k}, k=0,1,2, \ldots, M_{N}$. Note that monopoles in (b) and (c) are also distributed on a series of shells with radius $B=B_{k}$; and for the even case, there are no monopoles at the origin of the parameter space. netic fields two units of charges on the $k$ th shell are located at $\theta=0, \pi, B=B_{k}$ points; that is, all charges on each shell are carried by two monopoles located at the northern and southern poles of shells. So the density profile is a chain of monopoles located at $\mathbf{B}= \pm B_{k} \mathbf{e}_{z}, k+0,1, \ldots, M_{N}$.

This structure, however, is not generic. As we will show in the next section in the presence of a field gradient, charges on each shell are carried by smooth structures of monosegments instead of monopoles.

(iii) On each shell of $B=B_{k}, k=0,1,2, \ldots, M_{N}$ as shown in Fig. 2, the $\mathbf{b}$ field has a new component along the $\theta$ direction. This is due to the level crossing or more precisely presence of different spin states in exact ground states. The new component represents a Berry's phase when a closed path in the parameter space crosses $B=B_{k}$ surfaces. We will come back to this point in the next subsection.

\section{B. Berry's phases I: Rotating fields}

When a magnetic field $\mathbf{B}$ with a given magnitude rotates around the $z$ axis, a correlated state of spin-one atoms evolves along a path $\mathcal{C}$ shown in Fig. 3(a). During each period the state acquires a many-body Berry's phase. Consider a rotating magnetic field $\mathbf{B}(t)=B \mathbf{n}(t)$ with $\mathbf{n}(t)$ $=[\cos \phi(t) \sin \theta, \sin \phi(t) \sin \theta, \cos \theta] ; \theta$ is a constant and $\phi$ $=2 \pi \Omega t$ is time dependent.

Previous results on connection fields indicate that Berry's phases of many-body states

$$
\Phi_{B}(\mathcal{C})=\oint_{C} A \cdot d \mathbf{B}
$$

depend on the magnitude of magnetic fields. Following calculations in Appendix C:

$$
\Phi_{B}=-2 \pi \cos \theta Q(B) .
$$

As shown in Eq. (37), at a given magnetic field which is much smaller than $B_{M_{N}}$, the Berry's phase is always strongly suppressed because of antiferromagnetic correlations. We summarize our results in Fig. 4.

The nonanalytical behavior of Berry's phases in rotating fields is consistent with an anisotropic distribution of monopoles discussed in the last section. Consider two infinitesimal paths $\mathcal{C}_{1,2}$ centered at and also oriented along the $z$ axis. $\mathcal{C}_{1}$ is slightly above $-B_{k} \mathbf{e}_{z}$ and $\mathcal{C}_{2}$ slightly below. The Berry's phases evaluated in this way are singular at points $-B_{k} \mathbf{e}_{z}$ and experience a jump 

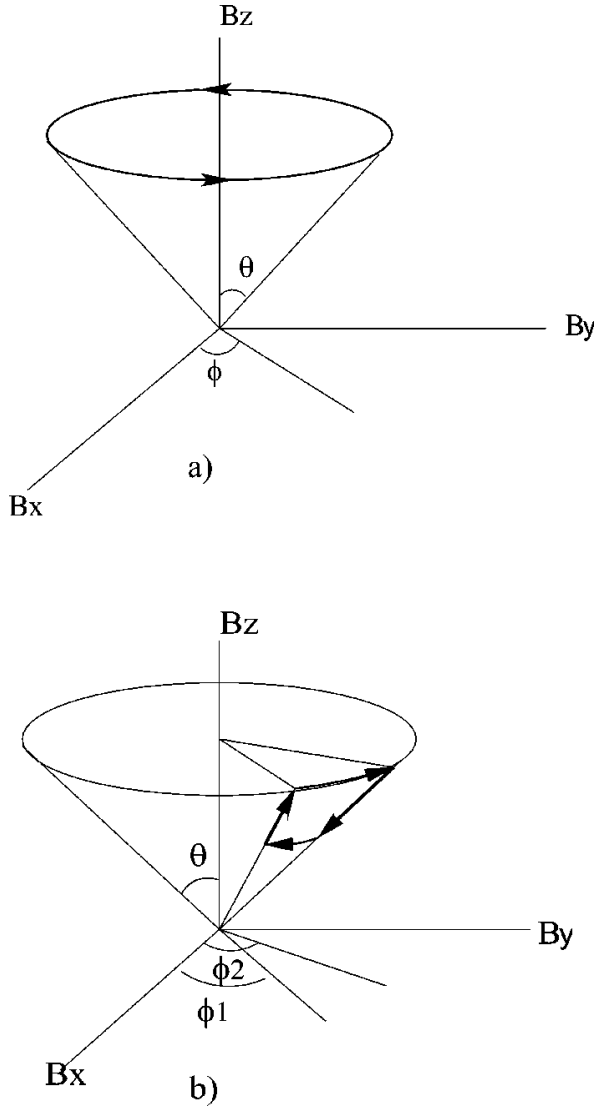

FIG. 3. Paths in a parameter space. (a) A path corresponding to a rotating field; (b) a path for a modulating field discussed in this subsection; the path is in $\theta=\theta_{0}$ surface.

$$
\Phi_{B}\left(\mathcal{C}_{2}\right)-\Phi_{B}\left(\mathcal{C}_{1}\right)=4 \pi
$$

Thus, the step-function plotted in Fig. 4 indeed implies that the surface bounded by path $C_{1}$ and $C_{2}$ enclose a monopole at $\mathbf{B}=-B_{k} \mathbf{e}_{z}$. One can apply similar argument at $\mathbf{B}=B_{k} \mathbf{e}_{z}$ and arrive at the same conclusions.

\section{Berry's phases II: Modulating fields}

For noninteracting cases, b fields only have $\mathbf{e}_{B}$ components. If a path completely lies in a plane parallel to $\mathbf{e}_{B}$, a quantum state does not acquire a Berry's phase because the topological flux threading the path should be zero for an obvious reason.

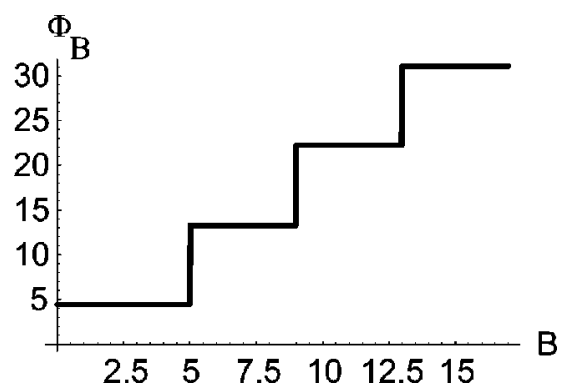

FIG. 4. A Berry's phase for a rotating field with $\theta=3 \pi / 4$ and various $B ; B$ is given in $g_{2} \gamma^{-1} . N$ is taken to be an odd number.
One of the interesting aspects of Eq. (29) is that there is a $\theta$ component in $\mathbf{b}$ fields because of antiferromagnetic interactions. This yields a new possibility to study Berry's phases which is uniquely associated with interacting particles. Consider a path that lies in a plane of $\theta=\theta_{0}$ and is bounded by

$$
B=B_{1,2}, \quad \phi=\phi_{1,2} .
$$

as shown in Fig. 3(b).

Following Eq. (29), one obtains

$$
\phi_{B}=-\cos \theta_{0}\left(\phi_{2}-\phi_{1}\right)\left[Q\left(B_{2}\right)-Q\left(B_{1}\right)\right] .
$$

This Berry's phase is nonzero only when $B=B_{1,2}$ surfaces are at two sides of $B=B_{k}$ surfaces. For $B_{k+1}>B_{2}>B_{k}>B_{1}>B_{k-1}$ and $\theta_{0}=3 \pi / 4, \phi_{B}=\left(\phi_{1}-\phi_{2}\right) \sqrt{2}$. The Berry's phase for modulating fields of this kind vanishes identically for noninteracting particles.

Discussions on geometrical phases are valid when slow spin relaxation is allowed so that the system can always reach true ground states in different magnetic fields within practically relevant time intervals. Furthermore, we also assume that the quantum symmetry restoring time is much shorter than measurement time; the issue of symmetry restoring of condensates of spin-one bosons was addressed in some detail in Ref. 34.

\section{MONOSEGMENTS AND BERRY'S PHASES OF $N$ INTERACTING SPIN-ONE BOSONS IN INHOMOGENEOUS MAGNETIC FIELDS}

\section{A. Spin conservation and magnetic field gradient}

In a homogeneous magnetic field, because of total spin conservation there is no mixing between states with different spins. However, a gradient in magnetic fields, as we will see does not conserve total spin and does mix states with different spins. This results in "level repulsion" when two spin states approach each other.

To show that an inhomogeneous magnetic field violates the conservation of total spin, we consider the commutator $\left[H, \hat{S}_{\text {tot }}^{2}\right]$ with $H$ given by

$$
\begin{gathered}
H=g_{2} \hat{\mathbf{S}}_{\mathrm{tot}}^{2}+\gamma \int d \mathbf{x} \mathbf{B}(\mathbf{x}) \cdot \hat{\psi}_{\alpha}^{\dagger} \mathbf{S}_{\alpha \beta} \hat{\psi}_{\beta}, \\
\mathbf{S}_{\beta \gamma}^{\alpha}=-i \epsilon_{\beta \gamma}^{\alpha} .
\end{gathered}
$$

One easily verifies that

$$
\begin{aligned}
{\left[\int d \mathbf{x}\right.} & \left.\mathbf{B}(\mathbf{x}) \cdot \hat{\psi}_{\alpha}^{\dagger} \mathbf{S}_{\alpha \beta} \hat{\psi}_{\beta}, \hat{\mathbf{S}}_{\mathrm{tot}}^{2}\right] \\
= & (2 i)\left[\int d \mathbf{x} \mathbf{B}(\mathbf{x}) \times \hat{\psi}_{\beta^{\dagger}}^{\dagger} \mathbf{S}_{\beta \beta^{\prime}} \hat{\psi}_{\beta}\right] \\
\times & {\left[\int d \mathbf{y} \hat{\psi}_{\alpha}^{\dagger}(\mathbf{y}) \mathbf{S}_{\alpha \alpha^{\prime}} \hat{\psi}_{\alpha^{\prime}}(\mathbf{y})\right] } \\
& -2 \int d \mathbf{x} \hat{\psi}_{\alpha}^{\dagger}(\mathbf{x}) \mathbf{B}(\mathbf{x}) \cdot \mathbf{S}_{\alpha \beta} \hat{\psi}_{\beta}(\mathbf{x})
\end{aligned}
$$

This commutator only vanishes in a homogeneous magnetic 
field but is nonzero when a field gradient is present(see Appendix B).

\section{B. Mixing of many-body states with different total spins}

For spatially varying magnetic fields $\mathbf{B}(\mathbf{x})$, we define creation and annihilation operators $\hat{\psi}_{\alpha}^{\prime}, \hat{\psi}_{\alpha}^{\prime \dagger}$ in a local triad where the local magnetic field points at the $z$ direction; the local field operators can be obtained by the following spin rotation:

$$
\hat{\psi}_{\alpha}^{\prime}(\mathbf{x})=U_{\alpha \beta}(\mathbf{x}) \hat{\psi}_{\beta}(\mathbf{x}),
$$

with $U_{\alpha \beta}(\mathbf{x})$ given by

$$
U_{\alpha \beta}(\mathbf{x})=\exp \left[i \boldsymbol{\theta}(\mathbf{x}) \cdot \mathbf{S}_{\alpha \beta}\right] .
$$

The Zeeman splitting in terms of spin-rotated operators is

$$
H_{\text {Zeeman }}=\gamma \int d \mathbf{x} B(\mathbf{x}) \hat{\psi}_{\alpha}^{\prime \dagger} S_{\alpha \beta}^{z} \hat{\psi}_{\beta}^{\prime}, S_{\alpha \beta}^{z}=-i \boldsymbol{\epsilon}_{\alpha \beta}^{z},
$$

with $B(\mathbf{x})=|\mathbf{B}(\mathbf{x})|$. On rotated spin-one fields, external magnetic fields always act along the $z$ axis.

One also finds that the spin-dependent and spinindependent interaction terms are invariant under the local spin rotation. However, the kinetic energy transforms nontrivially and acquires a term, $H_{\text {kin }} \rightarrow H_{\text {kin }}+H_{1}$ (see Appendix C).

Consider a field distribution

$$
\mathbf{B}(\mathbf{x})=B_{0}\left(1-G^{\prime} z\right) \hat{\mathbf{z}}+G^{\prime} B_{0} x \hat{\mathbf{x}} .
$$

We assume that $G^{\prime}$ is very small compared to the dimension of the system, i.e., $G^{\prime} \Omega^{1 / 3} \ll 1$. This ansatz was employed for the study of field gradient effects. ${ }^{26} \boldsymbol{\theta}$, which is determined by the relative orientation of magnetic fields with respect to the $z$ axis, can be calculated as

$$
\boldsymbol{\theta}(\mathbf{x})=\hat{\mathbf{z}} \times \hat{\mathbf{B}}(\mathbf{x})=G^{\prime} x \hat{\mathbf{y}} .
$$

Following Appendix $\mathrm{C}$, we find that the spin rotation considered above effectively orients external fields along the $z$ axis and results in a term $H_{1}$ in the single mode Hamiltonian, i.e.:

$$
H[\mathbf{B}(\mathbf{x})] \rightarrow H\left[B(\mathbf{x}) \mathbf{e}_{z}\right]+H_{1}
$$

and

$$
H_{1}=\frac{\epsilon}{2} \sum_{\alpha \neq y} \hat{\psi}_{\alpha}^{\dagger} \hat{\psi}_{\alpha}
$$

where $\epsilon=\hbar^{2} G^{\prime 2} / m$.

The tunneling matrix elements can be calculated as

$$
\left\langle 1,-1\left|\hat{H}_{1}\right| 3,-3\right\rangle=\frac{\epsilon}{2} \sqrt{\frac{3}{70}} \sqrt{(N-1)(N+4)} \stackrel{N \rightarrow \infty}{=} \frac{\epsilon}{2} \sqrt{\frac{3}{70}} N .
$$

For general level crossings in ground states, we obtain the following matrix elements. For an odd $N$ :

$$
\begin{aligned}
\Delta_{k} & =\left\langle(2 k+1),-(2 k+1)\left|\hat{H}_{1}\right|(2 k+3),-(2 k+3)\right\rangle \\
& =\frac{\hbar^{2} G^{\prime 2}}{4 m} \sqrt{\frac{(2 k+2)(N-2 k-1)(2 k+3)(N+2 k+4)}{(4 k+5)(4 k+7)}} .
\end{aligned}
$$

And for an even $N$ :

$$
\begin{aligned}
\Delta_{k} & =\left\langle 2 k,-2 k\left|\hat{H}_{1}\right| 2 k+2,-2 k-2\right\rangle \\
& =\frac{\hbar^{2} G^{\prime 2}}{4 m} \sqrt{\frac{(2 k+1)(N-2 k)(2 k+2)(N+2 k+3)}{(4 k+3)(4 k+5)}} .
\end{aligned}
$$

Therefore, a field gradient in magnetic fields has nonvanishing matrix elements between states with different spins and leads to mixing of corresponding many-body states. We will study the resultant spectrum in the next subsection.

\section{An effective Hamiltonian close to crossing points $B_{k}$}

For values of $|\mathbf{B}|$ close to the level crossing points $B_{k}$, we have a small tunneling term calculated earlier. If two nearly degenerate states are far away from other levels, the Hilbert space can be truncated into a two-level Hilbert space and the effective Hamiltonian in this subspace can be easily determined.

When $B$ is close to $B_{k}$, we therefore obtain an effective Hamiltonian in the truncated space

$$
H=\left[\begin{array}{cc}
E_{S_{k}, S_{k}}(B) & \Delta_{k} \\
\Delta_{k} & E_{S_{k}+2,-S_{k}-2}(B)
\end{array}\right] .
$$

$S_{k}=2 k+1$ for an odd $N$ and $S_{k}=2 k$ for an even $N$. And once again, $k=0,1,2, \ldots, M_{N}$. We have assumed that the gradient is small and $\Delta_{k} \ll g_{2}$ so that the truncation discussed here is always applicable when $B$ is close to $B_{k}$.

For two levels $|1,-1\rangle$ and $|3,-3\rangle$ at $B \sim B_{0}=5 g_{2} / \gamma$ :

$$
H=\left[\begin{array}{cc}
E_{1,-1}(B) & \Delta_{0} \\
\Delta_{0} & E_{3,-3}(B)
\end{array}\right]
$$

with

$$
\Delta_{0}=\sqrt{\frac{3}{70}} \frac{\hbar^{2} G^{\prime 2}}{2 m} N
$$

$E_{1,-1}=E_{3,-3}=E_{0}$ with $E_{0}=-3 g_{2}$ when $B=B_{0}$. Introduce $\delta B=B-B_{0}, \quad \delta E_{l,-l}=E_{l,-l}-E_{0}$. One obtains $\delta E_{1,-1}=-\gamma \delta B$, $\delta E_{3,-3}=-3 \gamma \delta B$. The eigenvalues can be expressed as

$$
E_{ \pm}(\delta B)=-3 g_{2}-2 \gamma \delta B \pm\left(\gamma^{2} \delta B^{2}+\Delta_{0}^{2}\right)^{1 / 2} .
$$

\section{Connection fields of spin mixed many-body states}

Because of the field gradient, matrix elements of the Hamiltonian between states of different spins are nonzero. Eigenstates are generally superpositions of states with different spins. 
Close to level crossing points $B_{k}$, in the truncated Hilbert space the eigenstates are

$$
\begin{gathered}
\Psi^{ \pm}(\mathbf{n})=\delta_{k 1}^{ \pm}\left|S_{k},-S_{k} ; \mathbf{n}\right\rangle+\delta_{k 2}^{ \pm}\left|S_{k}+2,-S_{k}-2 ; \mathbf{n}\right\rangle, \\
\left|\delta_{k 1}^{ \pm}\right|^{2}+\left|\delta_{k 2}^{ \pm}\right|^{2}=1 ;
\end{gathered}
$$

the corresponding many-body microscopic wave functions are

$$
\begin{aligned}
\Psi^{ \pm}(\mathbf{n})= & \delta_{k 1}^{ \pm} C\left(\hat{\psi}_{-1}^{\prime \prime \dagger}\right)^{S_{k}}\left(\hat{A}^{\dagger}\right)^{\left(N-S_{k}\right) / 2}|0\rangle \\
& +\delta_{k 2}^{ \pm} D\left(\psi_{-1}^{\prime \prime \dagger}\right)^{S_{k}+2}\left(\hat{A}^{\dagger}\right)^{\left(N-S_{k}-2\right) / 2}|0\rangle,
\end{aligned}
$$

where $C$ and $D$ are normalization constants. $\hat{\psi}_{-1}^{\prime \prime+}$ is a creation operator defined in a local frame discussed in Appendix C 1. $\Psi^{+}$refers to the higher energy state in the truncated Hilbert space and $\Psi^{-}$refers to the lower energy state. For a given $k$ :

$$
\begin{gathered}
S_{k}=2 k+1 \quad \text { for an odd } N, \\
S_{k}=2 k \text { for an even } N .
\end{gathered}
$$

Finally, as the magnetic field increases from below $B_{k}$ to above $B_{k}, \delta_{k 1}^{-}$varies from one to zero and $\delta_{k 2}^{-}$from zero to one. Taking into account the effective Hamiltonian in the truncated Hilbert space derived in Sec. IV C, we obtain the following field dependence of the coefficients $\delta_{k 1, k 2}^{ \pm}$when $B$ is close to $B_{k}$ :

$$
\begin{aligned}
& \delta_{k 1}^{ \pm}=\frac{\left(\gamma \delta B_{k} \pm \sqrt{\Delta_{k}^{2}+\gamma^{2} \delta B_{k}^{2}}\right)}{\sqrt{2\left(\gamma^{2} \delta B_{k}^{2}+\Delta_{k}^{2} \pm \gamma \delta B_{k} \sqrt{\Delta_{k}^{2}+\gamma^{2} \delta B_{k}^{2}}\right)}}, \\
& \delta_{k 2}^{ \pm}=\frac{\Delta_{k}}{\sqrt{2\left(\gamma^{2} \delta B_{k}^{2}+\Delta_{k}^{2} \pm \gamma \delta B_{k} \sqrt{\Delta_{k}^{2}+\gamma^{2} \delta B_{k}^{2}}\right)}} .
\end{aligned}
$$

In Eq. (60), we have introduced $\delta B_{k}=B-B_{k} . \Delta_{k} \quad(k$ $\left.=0,1,2, \ldots, M_{N}\right)$ represent the matrix elements calculated in Eq. (51); $\Delta_{k}=\left\langle(2 k+1),-(2 k+1)\left|\hat{H}_{1}\right|(2 k+3),-(2 k+3)\right\rangle$ for an odd $N$ and $\Delta_{k}=\left\langle(2 k),-(2 k)\left|\hat{H}_{1}\right|(2 k+2),-(2 k+2)\right\rangle$ for an even $N$.

Discussions on connection fields and topological charge densities can be carried out, similar to those in the previous section. After some straightforward calculations, we find

$$
\mathbf{A}=-\frac{\cos \theta}{\sin \theta} \frac{Q_{g}(|\mathbf{B}|)}{|\mathbf{B}|} \mathbf{e}_{\phi} .
$$

Assuming the field gradient is small, we find that $Q_{g}(|\mathbf{B}|)$ is identical to $Q(|\mathbf{B}|)$ defined in Sec. III A when $B$ is far away from degeneracy points $B_{k}$; close to $B_{k}$ when $\gamma\left|B-B_{k}\right|$ are comparable to $\Delta_{k}$, however:

$$
Q_{g}(|\mathbf{B}|)=S_{k}+2\left|\delta_{k 2}^{-}\right|^{2},
$$

which varies smoothly from $S_{k}$ to $S_{k}+2$. We want to emphasize that both $\delta_{k 1}^{-}$and $\delta_{k 2}^{-}$are real functions of $|\mathbf{B}|$ and independent of $\theta$ and $\phi$.

Following discussions in the previous sections, one obtains the two-form connection fields $\mathbf{F}_{a b}$ or $\mathbf{b}$ :

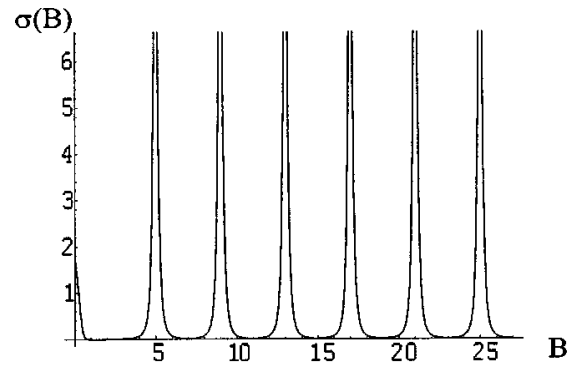

a)

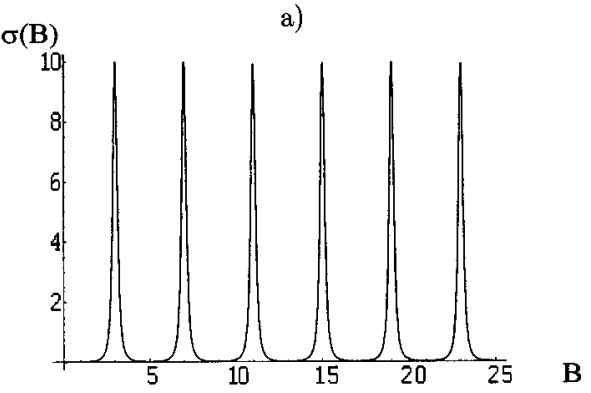

b)

FIG. 5. The surface charge $\sigma(B)$ as a function of magnetic field in the presence of a field gradient $G^{\prime}=1.32 \mathrm{~cm}^{-1} . B$ is measured in units of $g_{2} \gamma^{-1}$. (a) is for an odd number of particles and (b) for an even number of particles.

$$
\mathbf{b}=\frac{Q_{g}(|\mathbf{B}|)}{|\mathbf{B}|^{2}} \mathbf{e}_{B}+\frac{1}{B} \frac{\partial Q_{g}(B)}{\partial B} \frac{\cos \theta}{\sin \theta} \mathbf{e}_{\theta} .
$$

The topological charge density is

$$
\rho=q_{0}(N) \delta(\mathbf{B})+\frac{1}{2 \pi|\mathbf{B}|^{2} \sin \theta} \frac{\partial Q_{g}(|\mathbf{B}|)}{\partial B}[\delta(\theta-\pi)+\delta(\theta)] .
$$

Correspondingly, the surface charge becomes

$$
\sigma(B)=q_{0}(N) \delta(B)+\frac{\partial Q_{g}(B)}{\partial B},
$$

which is analytical at $B_{k}, k=0,1, \ldots, M_{N}$ because of level repulsion. Following Eqs. (60) and (62), far away from $B_{k}$, $\sigma(B)$ is vanishingly small. $\sigma(B)$ as a function of $B$ is numerically plotted in Fig. 5.

Equations (63) and (65) again clearly indicate shell structures. Indeed, when the width of each shell $2 h$ defined before Eq. (34) is much larger than a characteristic width $W_{k}$ :

$$
2 h \gg W_{k}=\frac{\Delta_{k}}{\gamma},
$$

the charge $Q_{k}$ enclosed in the $k$ th shell centered at $B=B_{k}$ is

$$
Q_{k}=2+O\left(\frac{W_{k}^{2}}{h^{2}}\right)
$$

$Q_{k}$ approaches two units when the ratio between $2 h$ and $W_{k}$ becomes infinity. And only on these shells, $\mathbf{b}$ fields have a $\theta$ component. 


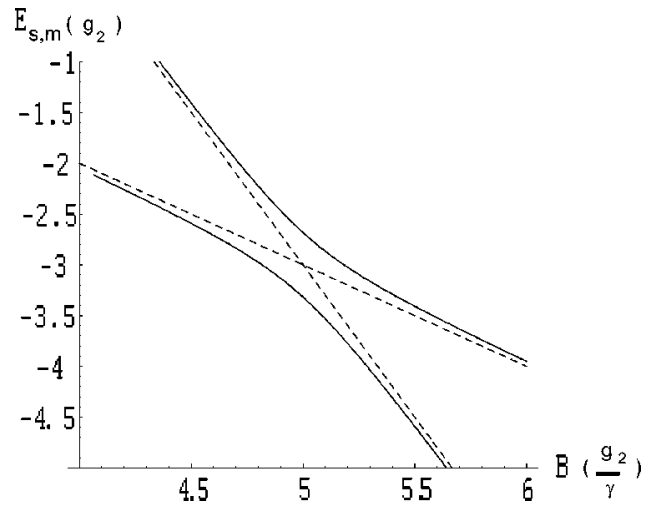

FIG. 6. Eigenvalues as functions of magnetic fields close to $B_{0}$; $G^{\prime}=1.32 \mathrm{~cm}^{-1}$.

Therefore, Eq. (64) shows that on each shell the charge distribution is highly anisotropic. All charges on each shell in this case are carried by two monosegments. Each monosegment carries one unit of charge and is centered at $\mathbf{B}$ $= \pm B_{k} \mathbf{e}_{z}$; a monosegment is smooth along the $z$ axis, the typical width of a monosegment located at $\mathbf{B}= \pm B_{k} \mathbf{e}_{z}$ is approximately $W_{k}$. Topological charges overall distribute in a chain of monosegments instead of monopoles. Finally, in the presence of field gradients, results in Eq. (35) are still valid.

Before we leave this subsection, we emphasize that $\mathbf{b}$ fields are gauge invariant under a usual $U(1)$ gauge transformation. Though this is hardly surprising by virtue of the two-form construction, in Appendix E we nevertheless present an explicit calculation to illustrate this point in terms of many-body wave functions.

\section{E. Landau-Zener effects}

$B(t)$ varies adiabatically. When $t \rightarrow-\infty$, the ground state is $|1,-1\rangle$ and when $t \rightarrow+\infty$ the ground state is $|3,-3\rangle$. Since $B$ changes adiabatically, for the most of time the system remains in the ground state; however, as the change rate is finite, the system also makes transitions to an excited state $|1,-1\rangle$ at $t \rightarrow \infty$ with small probability.

Denoting the excited state by + and the groundstate by -, the transition probability can be calculated with the following formula: ${ }^{37}$

$$
W_{+;-}=\exp \left(-2 \operatorname{Im} \int_{C}^{\tau_{0}} d \tau\left[E_{+}(\tau)-E_{-}(\tau)\right]\right) .
$$

Here $\tau_{0}$ is the complex value at which $E_{+}(\tau)=E_{-}(\tau)$ and $C$ a curve from $t \rightarrow-\infty$ to $t \rightarrow+\infty$ passing above $\tau_{0}$. Substituting the results found in Eq. (59) into the earlier expression, we get

$$
W_{+;-}=\exp \left[-8 \operatorname{Im} \int_{0}^{i \frac{\Delta_{0}}{v \gamma}} d \tau \sqrt{\Delta_{0}^{2}+\gamma^{2} v^{2} \tau^{2}}\right]=\exp \left[-2 \frac{\pi}{\gamma v} \Delta_{0}^{2}\right]
$$

with $v$ as the rate. In Fig. 6, we show the energy of these two levels as a function of magnetic field in the vicinity of $B_{0}$.
The steps in the Berry's phases as a function of magnetic field become rounded because of level repulsion.

\section{LARGE $N$ LIMIT}

\section{A. An effective Hamiltonian in large $N$ limit}

As proposed in a few previous works, the problem of interacting spin-one bosons can be mapped into a constrained quantum rotor model in the large $N$ limit. $^{27,28,31,32,34}$ And any microsopic many-body state can be expressed in terms of a wave function $\psi(\mathbf{n})$ of a quantum rotor characterized by its direction $\mathbf{n}$. In fact, an arbitrary wave function $\psi(\mathbf{n})$ represents the following microscopic wave function of a correlated state

$$
\Psi=\int d \mathbf{n} \psi(\mathbf{n})|\mathbf{n}\rangle ;|\mathbf{n}\rangle=\sqrt{\frac{N+1}{2 N !}}\left(\mathbf{n}_{\alpha} \hat{\psi}_{\alpha}^{\dagger}\right)^{N}|0\rangle .
$$

Therefore, a state where $\mathbf{n}$ is localized on a two sphere corresponds to a polar condensate; an $S$ wave of $\mathbf{n}$ represents a rotationally invariant spin singlet ground state. More discussions about connections between the two representations can be found in Ref. 34 .

The Hamiltonian for spin-one bosons in $|\mathbf{n}\rangle$-representation is given by a quantum rotor model

$$
H=g_{2} \hat{\mathbf{L}}^{2}+\gamma \mathbf{B} \cdot \hat{\mathbf{L}} \text {. }
$$

The total spin of spin-one particles $\hat{\mathbf{S}}_{\text {tot }}(=\hat{\mathbf{L}})$ is a differential operator

$$
\hat{\mathbf{L}}=-i \mathbf{n} \times \frac{\partial}{\partial \mathbf{n}}
$$

i.e., the total spin operator is the angular momentum operator defined on the two-sphere where $\mathbf{n}$ lives; $\hat{\mathbf{L}}$ is also a conjugate variable of $\mathbf{n}$. Wave functions further observe the following Ising symmetry:

$$
\psi(\mathbf{n})=(-1)^{N} \psi(-\mathbf{n})
$$

for any $N$; this property of many-body wave functions was identified and emphasized in previous works on homogeneous gases of spin-one bosons ${ }^{27,28}$ and on spin-one bosons in lattices. ${ }^{29,31,32,34}$

A direct calculation in Appendix $\mathrm{F}$ indicates the following relation in the $|\mathbf{n}\rangle$-representation

$$
\hat{\psi}_{\alpha}^{\dagger} h_{\alpha \beta} \hat{\psi}_{\beta} \rightarrow N \mathbf{n}_{\alpha} h_{\alpha \beta} \mathbf{n}_{\beta} .
$$

Therefore, the field gradient induced quadratic Zeeman term discussed in Appendix D results in the following coupling term in the quantum rotor representation (up to a shift)

$$
H_{1}=N \frac{\hbar^{2}}{2 m} h_{\alpha \beta} Q_{\alpha \beta} \text {. }
$$

Here

$$
h_{\alpha \beta}(\mathbf{r})=\nabla \theta_{\eta} \cdot \nabla \theta_{\xi} \mathbf{S}_{\alpha \gamma}^{\eta} \mathbf{S}_{\gamma \beta}^{\xi} ;
$$

and $Q_{\alpha \beta}$ is the nematic order parameter 


$$
Q_{\alpha \beta}=\mathbf{n}_{\alpha} \mathbf{n}_{\beta}-\frac{1}{3} \delta_{\alpha \beta} .
$$

For a magnetic field distribution given in this article, the $h$ matrix is

$$
h_{\alpha \beta}=G^{\prime 2}\left(\mathbf{S}^{y} \mathbf{S}^{y}\right)_{\alpha \beta} ;
$$

therefore, the effective Hamiltonian for the gradient term in the large $N$ limit is

$$
H_{1}=\frac{\hbar^{2} G^{\prime 2}}{2 m} N \sum_{\alpha \neq y} \mathbf{n}_{\alpha}^{2},
$$

which was also proposed in an early paper. ${ }^{28}$

\section{B. Effects due to anisotropy}

Taking into account anisotropy, the quantum rotor effective hamiltonian for a magnetic field distribution given in Eq. (46) is

$$
\begin{gathered}
H=g_{2} \hat{\mathbf{L}}^{2}+\gamma \mathbf{B} \cdot \hat{\mathbf{L}}+h_{\alpha \beta} Q_{\alpha \beta} N, \\
h_{\alpha \beta}=\frac{\hbar^{2} G^{\prime 2}}{2 m} \delta_{\alpha \beta}\left(\delta_{\alpha x}+\delta_{\alpha z}\right) .
\end{gathered}
$$

The tunneling term between different total spin states is given by

$$
\begin{gathered}
\left\langle s m\left|h_{\alpha \beta} \hat{\psi}_{\alpha}^{\dagger} \hat{\psi}_{\beta}\right| s^{\prime} m^{\prime}\right\rangle=h_{\alpha \beta} T_{\alpha \beta}\left(s m, s^{\prime} m^{\prime}\right) N, \\
T_{\alpha \beta}\left(s m, s^{\prime} m^{\prime}\right)=\int d \mathbf{n} Y_{s m}^{*}(\mathbf{n}) \mathbf{n}_{\alpha} \mathbf{n}_{\beta} Y_{s^{\prime} m^{\prime}}(\mathbf{n}) .
\end{gathered}
$$

For states $|1,-1\rangle$ and $|3,-3\rangle$, we have

$$
T_{\alpha \beta}(3,-3 ; 1,-1)=\sqrt{\frac{3}{70}}\left(\begin{array}{ccc}
1 & i & 0 \\
i & -1 & 0 \\
0 & 0 & 0
\end{array}\right) .
$$

And the matrix element between $|3,-3\rangle$ and $|1,-1\rangle$ is

$$
N h_{\alpha \beta} T_{\alpha \beta}(3,-3 ; 1,-1)=\frac{\hbar^{2} G^{\prime 2}}{2 m} \sqrt{\frac{3}{70}} N \text {. }
$$

Close to $B_{0}$, states $|1,-1\rangle$ and $|3,-3\rangle$ are nearly degenerate as mentioned before. In the two-level subspace, the Hamiltonian is identical to that in Eq. (53); $\Delta_{0}\left(=h_{\alpha \beta} T_{\alpha \beta}\right)$ is calculated earlier. One can then study the eigenstates in this subspace; after redefining $\Delta_{0}$, one obtains results identical to those in Sec. IV C.

\section{Berry's phases}

In the quantum rotor representation, the spectra of the Hamiltonian subject to Ising symmetries in Eq. (73) are identical to those discussed in Sec. II. The Berry's phases of many-body ground states in a rotating field can be evaluated in a straightforward way. All results in Secs. III and IV can be easily rederived; moreover, this effective description gives a simple geometric interpretation of results obtained in a microscopic calculation. We do not present detailed calculations here.

\section{Observation of Berry's phase}

The peculiar Berry's phases of many-body states might be observed by studying resonance transitions between a ground state and a collective excitation, similar to NMR experiments carried out earlier. ${ }^{5}$ In an adiabatically rotating magnetic field, the resonance frequency between these states should be shifted because of geometric phases. For instance, at $B$ less than $B_{0}$ the shift in the resonance frequency of transitions between an excited state $|2 k+1,-2 k-1\rangle$ and the ground state $|1,-1\rangle$ for an odd number of particles is

$$
\delta \omega=2 k \cos \theta \Omega .
$$

Here $\Omega$ is the rotating frequency and $\theta$ is the angle between the magnetic field and rotation axis $z$ (see Sec. III A for the geometry). Let us explain this in some detail.

\section{Adiabatically evolving states: General consideration}

Consider the following time-dependent Hamiltonian of a two level system

$$
H=\gamma \mathbf{B}(t) \cdot \frac{\sigma}{2} .
$$

The "magnetic field" $\mathbf{B}(t)$ is given by

$$
\begin{aligned}
\mathbf{B}(t)= & B \mathbf{n}(t)=B[\cos (\Omega t) \sin (\theta) \hat{\mathbf{x}}+\sin (\Omega t) \sin (\theta) \hat{\mathbf{y}} \\
& +\cos (\theta) \hat{\mathbf{z}}] .
\end{aligned}
$$

Let $| \pm(t)\rangle$ denote the instantaneous eigenstates of the Hamiltonian $H(t)$ :

$$
H(t)| \pm(t)\rangle=E^{ \pm}(t)| \pm(t)\rangle
$$

with $E^{ \pm}(t)= \pm \gamma B / 2$. These states acquire geometric phases when $H$ is time dependent. The corresponding states are

$$
\left|\psi_{-}(t)\right\rangle=e^{-(i / \hbar) E^{-} t} e^{-\int_{0}^{t}\left\langle-\left|\partial_{t}\right|-\right\rangle d t^{\prime}}|-(t)\rangle
$$

and

$$
\left|\psi_{+}(t)\right\rangle=e^{-(i / \hbar) E^{+} t} e^{-\int_{0}^{t} d t^{\prime}\left\langle+\left|\partial_{t}\right|+\right\rangle}|+(t)\rangle .
$$

The transition probability between $\left|\psi_{-}(t)\right\rangle$ and $\left|\psi_{+}(t)\right\rangle$ induced by a time-dependent field $\delta B^{z}(t)=\delta B_{0}^{z} \cos (\omega t)$ is

$$
P_{+-}(t)=\frac{4}{\hbar^{2}} \delta B_{0}^{z 2}\left|\left\langle+\left|\frac{\sigma^{z}}{4}\right|-\right\rangle\right| \frac{2\left|\sin \left(\frac{\tilde{E}^{+}-\tilde{E}^{-}}{2 \hbar} t-\frac{\omega}{2} t\right)\right|^{2}}{\left(\frac{\tilde{E}^{+}-\tilde{E}^{-}}{\hbar}-\omega\right)^{2}}
$$

with

$$
\widetilde{E}^{ \pm}=E^{ \pm}-\hbar \frac{\Omega}{2 \pi} \Phi_{B}^{ \pm}
$$

and 


$$
\Phi_{B}^{ \pm}=-\operatorname{Im} \oint d \phi\left\langle \pm\left|\partial_{\phi}\right| \pm\right\rangle
$$

The resonance frequency is given by

$$
\omega=\omega_{+-}=\frac{1}{\hbar}\left[E^{+}-E^{-}-\frac{\hbar \Omega}{2 \pi}\left(\Phi_{B}^{+}-\Phi_{B}^{-}\right)\right],
$$

which differs from the resonance frequency in a static field $\omega_{+-}^{s}=1 / \hbar\left(E^{+}-E^{-}\right)$.

\section{Shift in resonance frequencies for spin-one bosons in a magnetic field}

The resonance frequency between the levels $(3,-3)$ and $(1,-1)$ as a function of magnetic field is

$$
\omega_{(3,-3) ;(1,-1)}(B)=\frac{1}{\hbar}\left[E_{(3,-3)}-E_{(1,-1)}\right]-\frac{\Omega}{2 \pi}\left[\Phi_{B}^{(3,-3)}-\Phi_{B}^{(1,-1)}\right] .
$$

Here

$$
\Phi_{B}^{(1,-1)}=-\operatorname{Im} \oint d \phi\left\langle 1,-1\left|\partial_{\phi}\right| 1,-1\right\rangle
$$

and

$$
\Phi_{B}^{(3,-3)}=-\operatorname{Im} \oint d \phi\left\langle 3,-3\left|\partial_{\phi}\right| 3,-3\right\rangle .
$$

For odd $N$ when there is no magnetic field gradient present, the resonance frequency between the states $\left|2 k_{1}+1, m_{1}\right\rangle$ and $\left|2 k_{2}+1, m_{2}\right\rangle$ is

$$
\begin{aligned}
\omega_{\left(2 k_{1}+1, m_{1}\right) ;\left(2 k_{2}+1, m_{2}\right)}(B)= & \frac{1}{\hbar}\left(E_{2 k_{1}+1, m_{1}}-E_{2 k_{2}+1, m_{2}}\right) \\
& -\frac{\Omega}{2 \pi}\left[\Phi_{B}^{\left(2 k_{1}+1, m_{1}\right)}-\Phi_{B}^{\left(2 k_{2}+1, m_{2}\right)}\right],
\end{aligned}
$$

where

$$
\Phi_{B}^{(2 k+1, m)}=-\operatorname{Im} \oint d \phi\left\langle 2 k+1, m\left|\partial_{\phi}\right| 2 k+1, m\right\rangle .
$$

When there is a magnetic field gradient present (see Fig. 7), near the level crossing $B \approx B_{k}$ the low energy and high energy states are

$$
\Psi^{ \pm}(\mathbf{n})=\delta_{k 1}^{ \pm}\left|S_{k},-S_{k} ; \mathbf{n}\right\rangle+\delta_{k 2}^{ \pm}\left|S_{k}+2,-S_{k}-2 ; \mathbf{n}\right\rangle
$$

with coefficients specified in Eq. (60). Here we have that $\delta B_{k}=B-B_{k}$. Now the shifts in the resonance frequencies of the transitions between the states given in Eq. (99) are
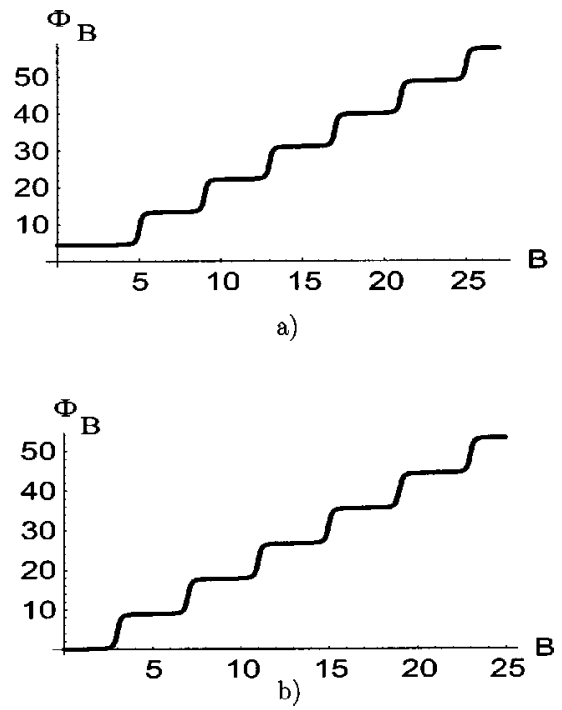

FIG. 7. Berry's phase in the presence of a field gradient $G^{\prime}$ $=1.32 \mathrm{~cm}^{-1}$. B is measured in units of $g_{2} \gamma^{-1}$. (a) is for an odd $N$. (b) is for an even $N$.

$$
\begin{aligned}
\omega_{+-, k}(B)= & \frac{1}{\hbar}\left(E_{k}^{+}-E_{k}^{-}\right)+\Omega \cos (\theta)\left\{\left[\left(\delta_{k, 1}^{+}\right)^{2}-\left(\delta_{k, 1}^{-}\right)^{2}\right] S_{k}\right. \\
& \left.+\left[\left(\delta_{k, 2}^{+}\right)^{2}-\left(\delta_{k, 2}^{-}\right)^{2}\right]\left(S_{k}+2\right)\right\} \\
= & \frac{1}{\hbar}\left(E_{k}^{+}-E_{k}^{-}\right)-2 \Omega \cos (\theta) \frac{\Delta E_{k}}{\sqrt{4 \Delta_{k}^{2}+\Delta E_{k}^{2}}} .
\end{aligned}
$$

Here

$$
E_{k}^{ \pm}=\frac{1}{2}\left[E_{(2 k+1),-(2 k+1)}+E_{(2 k+3),-(2 k+3)}\right] \pm \frac{1}{2} \sqrt{\Delta E_{k}^{2}+4 \Delta_{k}^{2}}
$$

and

$$
\Delta E_{k}=\left[E_{(2 k+1),-(2 k+1)}-E_{(2 k+3),-(2 k+3)}\right],
$$

where $E_{(2 k+1),-(2 k+1)}$ is the energy of a state $\mid 2 k+1,-(2 k$ $+1)\rangle$ and $\Delta_{k}$ is the matrix element between states $\mid 2 k+1$, $-(2 k+1)\rangle$ and $|2 k+3,-(2 k+3)\rangle$ as given in Secs. IV C and IV D. Eventually we find

$$
\omega_{+-, k}(B)=\frac{2}{\hbar}\left(\gamma^{2} \delta B_{k}^{2}+\Delta_{k}^{2}\right)^{1 / 2}-2 \Omega \cos \theta \frac{\gamma \delta B_{k}}{\left(\Delta_{k}^{2}+\gamma^{2} \delta B_{k}^{2}\right)^{1 / 2}}
$$

for the resonance frequency. The last term is the contribution from Berry's phases and $\gamma=g_{F} \mu_{B}$.

\section{CONCLUSION}

In this paper we discuss the Berry's connection fields of many-body states of spin-one bosons with antiferromagnetic interactions. We show that unlike noninteracting systems, Berry's connection fields are determined by a linear chain of monopoles; more over in the presence of field gradients each monopole becomes a linearly extended object which we call 
a monosegment. Antiferromagnetic interactions appear to result in anisotropic expulsion of topological charges from the origin of parameter space and suppression of Berry's phases in small rotating magnetic fields.

\section{ACKNOWLEDGMENTS}

One of the authors (F.Z.) wants to thank the ASPEN center for physics for its hospitality during the year 2003 ASPEN workshop on quantum gases. He also acknowledges a very pleasant conversation with $\mathrm{S}$. F. Su, L. T. Wang, and Q. Niu during which the notion of monoshell was suggested. This work was supported by the Foundation FOM, the Netherlands under Contract Nos. 00CCSPP10, 02SIC25, and NWO-MK "projectruimte” 00PR1929.

\section{APPENDIX A: BASIC ALGEBRAS}

One can verify the following algebra:

$$
\begin{gathered}
{\left[\hat{\mathbf{S}}^{\alpha}, \hat{\psi}_{\beta}\right]=i \epsilon^{\alpha \beta \gamma} \hat{\psi}_{\gamma},} \\
{\left[\hat{\mathbf{S}}^{\alpha}, \hat{\psi}_{\beta}^{\dagger}\right]=i \epsilon^{\alpha \beta \gamma} \hat{\psi}_{\gamma}^{\dagger},} \\
{\left[\hat{\mathbf{S}}^{\alpha}, \hat{\mathbf{S}}^{\beta}\right]=i \epsilon^{\alpha \beta \gamma} \hat{\mathbf{S}}^{\gamma},} \\
{\left[\hat{\rho}, \hat{\psi}_{\alpha}\right]=-\hat{\psi}_{\alpha},} \\
{\left[\hat{\rho}, \hat{\psi}_{\alpha}^{\dagger}\right]=\hat{\psi}_{\alpha}^{\dagger},} \\
{\left[\hat{\mathbf{S}}^{\alpha}, \hat{\rho}\right]=0 .}
\end{gathered}
$$

Furthermore, we define the singlet creation operator:

$$
\begin{aligned}
\hat{A}^{\dagger} & =\frac{1}{\sqrt{6}} \hat{\psi}_{\alpha}^{\dagger} \hat{\psi}_{\alpha}^{\dagger} \quad \text { with } \alpha=x, y, z \\
& =\frac{1}{\sqrt{6}}\left(\hat{\psi}_{0}^{\dagger} \hat{\psi}_{0}^{\dagger}-2 \hat{\psi}_{-1}^{\dagger} \hat{\psi}_{1}^{\dagger}\right) .
\end{aligned}
$$

This operator satisfies

$$
\left[\hat{A}, \hat{A}^{\dagger}\right]=\frac{1}{3}(3+2 \hat{N}) .
$$

\section{APPENDIX B: FIELD GRADIENT AND SPIN CONSERVATION}

In this appendix, we prove that a field gradient does not conserve the total spin of spin-one bosons. The second quantized form of the total spin operator $\hat{\mathbf{S}}_{\text {tot }}^{2}$ is given by

$$
\begin{aligned}
\hat{\mathbf{S}}_{\mathrm{tot}}^{2}= & 2 \int d \mathbf{x} \hat{\psi}_{\alpha}^{\dagger}(\mathbf{x}) \hat{\psi}_{\alpha}(\mathbf{x}) \\
& +\int d \mathbf{x} \int d \mathbf{y} \hat{\psi}_{\alpha}^{\dagger}(\mathbf{x}) \hat{\psi}_{\beta}^{\dagger}(\mathbf{y}) \mathbf{S}_{\alpha \alpha^{\prime}} \cdot \mathbf{S}_{\beta \beta^{\prime}} \hat{\psi}_{\beta^{\prime}}(\mathbf{y}) \hat{\psi}_{\alpha^{\prime}}(\mathbf{x}) .
\end{aligned}
$$

We consider the commutator

$$
\left[\hat{\mathbf{S}}_{\mathrm{tot}}^{2}, \gamma \int d \mathbf{x} \mathbf{B}(\mathbf{x}) \cdot \hat{\psi}_{\alpha}^{\dagger}(\mathbf{x}) \mathbf{S}_{\alpha \beta} \hat{\psi}_{\beta}(\mathbf{x})\right] .
$$

Calculations yield

$$
\begin{aligned}
{\left[\int d \mathbf{x}\right.} & \left.\mathbf{B}(\mathbf{x}) \cdot \hat{\psi}_{\alpha}^{\dagger} \mathbf{S}_{\alpha \beta} \hat{\psi}_{\beta}, \hat{\mathbf{S}}_{\mathrm{tot}}^{2}\right] \\
= & {\left[\int d \mathbf{x} \mathbf{B}(\mathbf{x}) \cdot \hat{\psi}_{\alpha}^{\dagger} \mathbf{S}_{\alpha \beta} \hat{\psi}_{\beta}, 2 \int d \mathbf{x} \hat{\psi}_{\alpha}^{\dagger}(\mathbf{x}) \hat{\psi}_{\alpha}(\mathbf{x})\right] } \\
+ & {\left[\int d \mathbf{x} \mathbf{B}(\mathbf{x}) \cdot \hat{\psi}_{\alpha}^{\dagger} \mathbf{S}_{\alpha \beta} \hat{\psi}_{\beta}, \int d \mathbf{x} \int d \mathbf{y} \hat{\psi}_{\alpha}^{\dagger}(\mathbf{x}) \hat{\psi}_{\beta}^{\dagger}(\mathbf{y})\right.} \\
& \left.\times \mathbf{S}_{\alpha \alpha^{\prime}} \cdot \mathbf{S}_{\beta \beta^{\prime}} \hat{\psi}_{\beta^{\prime}}(\mathbf{y}) \hat{\psi}_{\alpha^{\prime}}(\mathbf{x})\right] .
\end{aligned}
$$

Using

$$
[A, B C]=[A, B] C+B[A, C]
$$

and

$$
\left[\hat{\psi}_{\alpha}(\mathbf{y}), \hat{\psi}_{\beta}^{\dagger}(\mathbf{x})\right]=\delta_{\alpha \beta} \delta(\mathbf{x}-\mathbf{y})
$$

we find that the first term vanishes. The second term can be simplified using the relations above and in the end we find results in Eq. (43). Now take $\mathbf{B}(\mathbf{x})$ homogeneous. We find

$$
\begin{aligned}
{\left[\int d \mathbf{x}\right.} & \left.\mathbf{B}(\mathbf{x}) \cdot \hat{\psi}_{\alpha}^{\dagger} \mathbf{S}_{\alpha \beta} \hat{\psi}_{\beta}, \hat{\mathbf{S}}_{\mathrm{tot}}^{2}\right] \\
= & (-2 i) \mathbf{B} \cdot\left[\int d \mathbf{x} \hat{\psi}_{\beta}^{\dagger}(\mathbf{x}) \mathbf{S}_{\beta \beta^{\prime}} \hat{\psi}_{\beta^{\prime}}(\mathbf{x})\right] \\
& \times\left[\int d \mathbf{y} \hat{\psi}_{\alpha}^{\dagger}(\mathbf{y}) \mathbf{S}_{\alpha \alpha^{\prime}} \hat{\psi}_{\alpha^{\prime}}(\mathbf{y})\right] \\
& -2 \mathbf{B} \cdot \int d \mathbf{x} \hat{\psi}_{\alpha}^{\dagger}(\mathbf{x}) \mathbf{S}_{\alpha \beta} \hat{\psi}_{\beta}(\mathbf{x}) \\
= & (-2 i) \mathbf{B} \cdot\left(\hat{\mathbf{S}}_{\mathrm{tot}} \times \hat{\mathbf{S}}_{\mathrm{tot}}\right)-2 \mathbf{B} \cdot \hat{\mathbf{S}}_{\mathrm{tot}} \\
= & (-i) B_{i} \epsilon_{i j k}\left[\hat{S}_{j}^{\mathrm{tot}}, \hat{S}_{k}^{\mathrm{tot}}\right]-2 B^{i} \hat{S}_{i}^{\mathrm{tot}}=0 .
\end{aligned}
$$

So for a homogeneous magnetic field total spin is conserved and an inhomogeneous magnetic field breaks conservation of total spin.

\section{APPENDIX C: CALCULATIONS OF BERRY'S CONNECTION FIELDS}

\section{General discussions: Without field gradient}

Define a coordinate frame $\left(x^{\prime}, y^{\prime}, z^{\prime}\right)$ with the $z^{\prime}$ axis in the direction of the magnetic field $\mathbf{n}$ $=(\sin \theta \cos \phi, \sin \theta \sin \phi, \cos \theta)$, and the $x^{\prime}$ axis in the $(x, y)$ plane. The creation operator in the local frame is 


$$
\hat{\psi}_{-1}^{\prime \prime \dagger}=-i e^{-i \phi} \sin ^{2}\left(\frac{\theta}{2}\right) \hat{\psi}_{1}^{\dagger}+i \frac{\sin \theta}{\sqrt{2}} \hat{\psi}_{0}^{\dagger}-i \cos ^{2}\left(\frac{\theta}{2}\right) e^{i \phi} \hat{\psi}_{-1}^{\dagger} .
$$

Therefore, $\left\langle\Psi_{S,-S}(\mathbf{n}) \mid d \Psi_{S,-S}(\mathbf{n})\right\rangle$ is given by

$$
\begin{aligned}
& \left\langle\Psi_{S,-S}(\mathbf{n}) \mid d \Psi_{S,-S}(\mathbf{n})\right\rangle \\
& =C^{2} S\langle 0| \hat{A}^{(N-S) / 2} \hat{\psi}_{-1}^{\prime \prime S}\left[-e^{-i \phi} \sin ^{2}\left(\frac{\theta}{2}\right) \hat{\psi}_{1}^{\dagger}\right. \\
& \left.\quad+e^{i \phi} \cos ^{2}\left(\frac{\theta}{2}\right) \hat{\psi}_{-1}^{\dagger}\right] \\
& \quad \times\left(\hat{\psi}_{-1}^{\prime \prime \dagger}\right)^{S-1} \hat{A}^{\dagger(N-S) / 2}|0\rangle d \phi .
\end{aligned}
$$

The results for connection fields follow this identity. Now the Berry's phase for $N$ interacting spin-one atoms with total spin $S$ is given by

$$
\phi_{\text {Berry }}=\operatorname{Im} \oint_{C}\left\langle\Psi_{S-S}(n) \mid d \Psi_{S-S}(n)\right\rangle=-2 \pi S \cos (\theta) .
$$

\section{General discussions: With a field gradient}

Now for $B \sim B_{k}$ :

$$
\begin{aligned}
& \left\langle\Psi_{S,-S}(\mathbf{n}) \mid d \Psi_{S,-S}(\mathbf{n})\right\rangle \\
& =i \cos \theta\left\{C^{2} S_{k} \delta_{k 1}^{-2}(B) d \phi\left\langle 0\left|\hat{A}^{\left(N-S_{k}\right) / 2} \hat{\psi}_{-1}^{\prime \prime} S_{k} \hat{\psi}_{-1}^{\prime \prime \dagger} S_{k} \hat{A}^{\dagger\left(N-S_{k}\right) / 2}\right| 0\right\rangle\right. \\
& +\left(S_{k}+2\right) D^{2} \delta_{k 2}^{-2}(B) d \phi\langle 0| \hat{A}^{\left(N-S_{k}-2\right) / 2} \hat{\psi}_{-1}^{\prime \prime}\left(S_{k}+2\right) \hat{\psi}_{-1}^{\prime \prime \dagger\left(S_{k}+2\right)} \\
& \times \hat{A}^{\dagger N-S_{k}-2 / 2}|0\rangle+\left(S_{k}+2\right) \delta_{k 1}^{-}(B) \delta_{k 2}^{-}(B) C D d \phi \\
& \times\left\langle 0\left|\hat{A}^{\left(N-S_{k}\right) / 2} \psi_{-1}^{\prime \prime} S_{k} \hat{\psi}_{-1}^{\prime \prime \dagger\left(S_{k}+2\right)} \hat{A}^{\dagger\left(N-S_{k}-2\right) / 2}\right| 0\right\rangle \\
& +S_{k} \delta_{k 1}(B) \delta_{k 2}(B) C D d \phi \\
& \left.\times\left\langle 0\left|\hat{A}^{\left(N-S_{k}-2\right) / 2} \hat{\psi}_{-1}^{\prime \prime}\left(S_{k}+2\right) \hat{\psi}_{-1}^{\prime \prime}{ }^{\dagger} \hat{A}_{k}^{\dagger\left(N-S_{k}\right) / 2}\right| 0\right\rangle\right\} \\
& =+i\left[\delta_{k 1}^{-2}(B) S_{k}+\delta_{k 2}^{-2}(B)\left(S_{k}+2\right)\right] \cos \theta d \phi \text {. }
\end{aligned}
$$

\section{Berry's local connection fields and topological charge densities}

As mentioned in the introduction, Berry's connection fields of the ground state are given by

$$
\mathbf{A}(\mathbf{B})=-\operatorname{Im}\left\langle g\left|\frac{\partial}{\partial \mathbf{B}}\right| g\right\rangle .
$$

For a state $|S,-S\rangle$, following discussions in Appendix C 1 and $\mathrm{C} 2$, Berry's connection potentials in spherical coordinates $(\theta, \phi, B)$ are

$$
\mathbf{A}(\mathbf{B})=-\frac{Q(\mathbf{B})}{B} \cot \theta \mathbf{e}_{\phi} .
$$

Now the Berry's connection fields are given by:

$$
\begin{aligned}
\mathbf{b} & =\nabla_{\mathbf{B}} \times \mathbf{A}(\mathbf{B}) \\
& =\frac{Q(B)}{B^{2}} \mathbf{e}_{B}+\frac{1}{B} \frac{\partial Q(B)}{\partial B} \cot (\theta) \mathbf{e}_{\theta} .
\end{aligned}
$$

For $B \neq 0, \theta \neq \pi$, and $\theta \neq 0$, we find

$$
\begin{aligned}
4 \pi \rho= & \nabla_{\mathbf{B}} \cdot \mathbf{b}=\frac{1}{B^{2}} \frac{\partial}{\partial B}\left[B^{2} \frac{Q(B)}{B^{2}}\right] \\
& +\frac{1}{B \sin \theta} \frac{\partial}{\partial \theta}\left[\frac{1}{B} \frac{\partial Q(B)}{\partial B} \cos \theta\right] \\
= & \frac{1}{B^{2}} \frac{\partial Q(B)}{\partial B}-\frac{1}{B^{2}} \frac{\partial Q(B)}{\partial B}=0 .
\end{aligned}
$$

At $B=0$ or $\theta=\pi, \theta=0$, and $B=B_{k}$ points, the $b$ fields are singular. Calculations around those points lead to results in Eq. (31).

\section{APPENDIX D: QUADRATIC ZEEMAN EFFECTS DUE TO FIELD GRADIENTS}

We show explicitly for the invariance of a spin-dependent term in the interaction. Let $U_{\alpha \beta}=\exp \left[i \boldsymbol{\theta} \cdot \mathbf{S}_{\alpha \beta}\right]$ :

$$
\begin{aligned}
H_{\mathrm{int}}= & \frac{c_{2}}{2} \int d \mathbf{x} \hat{\psi}_{\alpha}^{\dagger} \hat{\psi}_{\beta}^{\dagger} \mathbf{S}_{\alpha \alpha^{\prime}} \cdot \mathbf{S}_{\beta \beta^{\prime}} \hat{\psi}_{\alpha^{\prime}} \hat{\psi}_{\beta^{\prime}} \\
= & \frac{c_{2}}{2} \int d \mathbf{x} \hat{\psi}_{\gamma}^{\prime \dagger} U_{\gamma \alpha}(\mathbf{x}) \hat{\psi}_{\delta}^{\prime \dagger} U_{\delta \beta}(\mathbf{x}) \mathbf{S}_{\alpha \alpha^{\prime}} \cdot \mathbf{S}_{\beta \beta^{\prime}} \\
& \times U_{\alpha^{\prime} \gamma^{\prime}}^{\dagger}(\mathbf{x}) \psi_{\gamma^{\prime}}^{\prime} U_{\beta^{\prime} \delta^{\prime}}^{\dagger}(\mathbf{x}) \psi_{\delta^{\prime}}^{\prime} .
\end{aligned}
$$

Now using

$$
\begin{aligned}
U_{\alpha \alpha^{\prime}} \mathbf{S}_{\alpha^{\prime} \beta^{\prime}} U_{\beta^{\prime} \beta}^{\dagger}= & \left(U_{\alpha \alpha^{\prime}} \hat{\mathbf{x}} \cdot \mathbf{S}_{\alpha^{\prime} \beta^{\prime}} U_{\beta^{\prime} \beta}^{\dagger}, U_{\alpha \alpha^{\prime}} \hat{\mathbf{y}} \cdot \mathbf{S}_{\alpha^{\prime} \beta^{\prime}}\right. \\
& \left.\times U_{\beta^{\prime} \beta}^{\dagger}, U_{\alpha \alpha^{\prime}} \hat{\mathbf{z}} \cdot \mathbf{S}_{\alpha^{\prime} \beta^{\prime}} U_{\beta^{\prime} \beta}^{\dagger}\right) \\
= & \left(\hat{\mathbf{x}}^{\prime} \cdot \mathbf{S}_{\alpha \beta}, \hat{\mathbf{y}}^{\prime} \cdot \mathbf{S}_{\alpha \beta}, \hat{\mathbf{z}}^{\prime} \cdot \mathbf{S}_{\alpha \beta}\right)
\end{aligned}
$$

where the primes correspond to the rotated coordinate system and the fact that the inner product is rotationally invariant we find that the spin-dependent interaction term is invariant under the local spin rotation.

Now the kinetic term

$$
H=\int d \mathbf{x}-\frac{\hbar^{2}}{2 m} \hat{\psi}_{\alpha}^{\dagger}(\mathbf{x}) \nabla^{2} \hat{\psi}_{\alpha}(\mathbf{x})
$$

becomes in terms of $\hat{\psi}_{\alpha}^{\prime \dagger}$ and $\hat{\psi}_{\beta}^{\prime}$ :

$$
\begin{aligned}
H= & \int d \mathbf{x} \hat{\psi}_{\delta}^{\prime \dagger} U_{\delta \epsilon}(\mathbf{x}) \nabla^{2} U_{\epsilon \alpha}^{\dagger}(\mathbf{x}) \hat{\psi}_{\alpha}^{\prime}(\mathbf{x}) \\
= & \int d \mathbf{x}-\frac{\hbar^{2}}{2 m} \hat{\psi}_{\alpha}^{\prime \dagger}(\mathbf{x}) \nabla^{2} \hat{\psi}_{\alpha}^{\prime \dagger}(\mathbf{x})+\int d \mathbf{x} \\
& -\frac{\hbar^{2}}{2 m} \hat{\psi}_{\alpha}^{\prime \dagger}(\mathbf{x}) U_{\alpha \beta}\left(\nabla U_{\beta \gamma}^{\dagger}\right) \cdot \nabla \hat{\psi}_{\gamma}^{\prime}(\mathbf{x})+\int d \mathbf{x}-\frac{\hbar^{2}}{2 m} \hat{\psi}_{\alpha}^{\prime \dagger}(\mathbf{x}) \\
& \times\left(U_{\alpha \beta}(\mathbf{x}) \nabla^{2} U_{\beta \gamma}^{\dagger}(\mathbf{x})\right) \hat{\psi}_{\gamma}^{\prime}(\mathbf{x}) .
\end{aligned}
$$


Using the relation

$$
\frac{\partial}{\partial x_{i}} \exp [i \boldsymbol{\theta}(\mathbf{x}) \cdot \hat{\mathbf{S}}]=i\left(\frac{\partial \boldsymbol{\theta}(\mathbf{x})}{\partial x_{i}} \cdot \hat{\mathbf{S}}\right) \exp [i \theta \cdot \hat{\mathbf{S}}]
$$

and

$$
\frac{\partial \boldsymbol{\theta}}{\partial x_{i}}=\delta_{i, x} G^{\prime} \hat{\mathbf{y}}
$$

for the ansatz in Eq. (54), we have $H=H_{0}+H_{1}$, and

$$
\begin{aligned}
H_{1}= & \frac{\hbar^{2}}{2 m} \int d \mathbf{x} \hat{\psi}_{\alpha}^{\prime \dagger} U_{\alpha \beta}\left(i G^{\prime} S^{y}\right)_{\beta \gamma} U_{\gamma \eta}^{\dagger} \frac{\partial}{\partial x} \hat{\psi}_{\eta}^{\prime} \\
& -\frac{\hbar^{2}}{2 m} \int d \mathbf{x} \hat{\psi}_{\alpha}^{\prime \dagger} U_{\alpha \beta}\left[-G^{\prime 2}\left(S^{y}\right)^{2}\right]_{\beta \gamma} U_{\gamma \eta}^{\dagger} \hat{\psi}_{\eta}^{\prime} .
\end{aligned}
$$

By keeping only the zero mode contributions, we find

$$
H_{1}=\frac{\hbar^{2} G^{\prime 2}}{2 m} \hat{\psi}_{\alpha}^{\dagger}\left(S^{y}\right)_{\alpha \beta}^{2} \hat{\psi}_{\beta}
$$

This is the effective quadratic coupling in the presence of a field gradient which was previously obtained in Ref. 26.

\section{APPENDIX E: GAUGE INVARIANCE OF TOPOLOGICAL \\ FIELDS}

\section{General}

We define the following basis:

$$
\begin{aligned}
|S,-S ; \mathbf{n}\rangle= & C(S) e^{i P(B, \theta, \phi)}\left[-i \sin ^{2}\left(\frac{\theta}{2}\right) e^{-i \phi} \hat{\psi}_{+1}^{\dagger}\right. \\
& \left.+\frac{i}{\sqrt{2}} \sin \theta \hat{\psi}_{0}^{\dagger}-i \cos ^{2}\left(\frac{\theta}{2}\right) e^{i \phi} \hat{\psi}_{-1}^{\dagger}\right]^{S} \\
& \times \hat{A}^{\dagger(N-S) / 2}|0\rangle .
\end{aligned}
$$

Close to $B_{k}$ points, ground states are in general superpositions of these states; using the ansatz introduced in Sec. IV D, we find

$$
\begin{aligned}
\Psi(B, \mathbf{n})= & \delta_{k 1}(B)\left|S_{k},-S_{k} ; \mathbf{n}\right\rangle \\
& +\delta_{k 2}(B) e^{i d_{k}(\theta, \phi, B)}\left|S_{k}+2,-S_{k}-2 ; \mathbf{n}\right\rangle
\end{aligned}
$$

with $\delta_{k 1, k 2}, d_{k}$ functions of $(B, \phi, \theta)$. Calculations yield $\delta_{k 1, k 2}$ as given in Sec. IV D; $d_{k}=0$.
Therefore, we find

$$
\mathbf{A}(\mathbf{B})=-\frac{\partial P}{\partial B} \mathbf{e}_{B}-\frac{1}{B} \frac{\partial P}{\partial \theta} \mathbf{e}_{\theta}-\frac{1}{B \sin \theta}\left[\frac{\partial P}{\partial \phi}+Q_{g}(B) \cos \theta\right] \mathbf{e}_{\phi} .
$$

Note that Berry's connection potentials $\mathbf{A}(\mathbf{B})$ transform in an expected form

$$
\mathbf{A}(\mathbf{B}) \rightarrow \mathbf{A}(\mathbf{B})+\nabla \delta P(B, \theta, \phi),
$$

under a gauge transformtion $P \rightarrow P+\delta P$ :

$$
\mathbf{b}(\mathbf{B})=\frac{Q_{g}(B)}{B^{2}} \mathbf{e}_{B}+\frac{1}{B} \frac{\partial Q_{g}(B)}{\partial B} \frac{\cos \theta}{\sin \theta} \mathbf{e}_{\theta} .
$$

We find on the $k$ th shell two monopoles, one at the southern and one at the northern pole. The connection field $\mathbf{b}(\mathbf{B})$ does not depend on the choice of $P(B, \theta, \phi)$, i.e., the gauge.

\section{Case $P(B, \theta, \phi)=0$}

We find in this limit

$$
\mathbf{A}(\mathbf{B})=-\frac{\cos \theta}{B \sin \theta} Q_{g}(B) \mathbf{e}_{\phi}
$$

$$
\mathbf{b}(\mathbf{B})=\frac{Q_{g}(B)}{B^{2}} \mathbf{e}_{B}+\frac{1}{B} \frac{\partial Q_{g}(B)}{\partial B} \frac{\cos \theta}{\sin \theta} \mathbf{e}_{\theta} .
$$

\section{APPENDIX F: ESTIMATE FOR COLD SODIUM ATOMS}

Consider a trap with $N=10^{6}$ atoms, a density $n$ $=10^{14} \mathrm{~cm}^{-3}$ and $a_{2}-a_{0}=0.32 \mathrm{~nm}$. We have $g_{F}=-\frac{1}{2}$ for ${ }^{23} \mathrm{Na}$. The Zeeman effect is characterized by $\gamma$ :

$$
\gamma=g_{F} \mu_{B}=-3.3585 \times 10^{-5} \frac{K}{G} .
$$

Note that $\gamma$ is negative. Previous results can be applied provided we take for the magnetic field $\mathbf{B}=-B \mathbf{n}(\theta, \phi)$. One also finds

$$
\Delta_{0}=2.18 \times 10^{-15} \mathrm{Km}^{2} \mathrm{G}^{\prime 2},
$$

where we have the following units: $G^{\prime}$ in $\mathrm{m}^{-1}$ and $\Delta$ in $\mathrm{K}$. Furthermore

$$
g_{2}=10^{-15} \mathrm{~K} \text {. }
$$

The eigenvalues of $H$ in the vicinity of $B_{0}$ are 


$$
\begin{aligned}
E^{ \pm}(\delta B)= & -3 g_{2} \\
& -2 \gamma \delta B \pm \gamma \sqrt{4.23 \times 10^{-21} G^{2} m^{4} G^{\prime 4}+(\delta B)^{2}} .
\end{aligned}
$$

The relative level repulsion $\Delta E / g_{2}$ is $\sim 4 m^{2} G^{\prime 2}$ with $G^{\prime}$ in $\mathrm{m}^{-1}$.

\section{APPENDIX G: CALCULATION OF MATRIX ELEMENTS IN A LARGE- $N$ LIMIT}

Now the general operator $\hat{\psi}_{\alpha}^{\dagger} h_{\alpha \beta} \hat{\psi}_{\beta}$ with $h_{\alpha \beta}$ some matrix acts on the wave function as follows:

$$
\begin{aligned}
&\left\langle\psi_{1}(\mathbf{n})\left|\hat{\psi}_{\alpha}^{\dagger} h_{\alpha \beta} \hat{\psi}_{\beta}\right| \psi_{2}(\mathbf{n})\right\rangle \\
&=\int d \mathbf{n}_{1} \int d \mathbf{n}_{2} \frac{N+1}{2 N !}\langle 0|\left(n_{\gamma}^{1} \hat{\psi}_{\gamma}\right)^{N} \\
& \quad \times \hat{\psi}_{\alpha}^{\dagger} h_{\alpha \beta} \hat{\psi}_{\beta}\left(n_{\delta}^{2} \hat{\psi}_{\delta}^{\dagger}\right)^{N}|0\rangle \psi_{1}^{*}\left(\mathbf{n}_{1}\right) \psi_{2}\left(\mathbf{n}_{2}\right) \\
&=\int d \mathbf{n}_{1} \int d \mathbf{n}_{2} \frac{N+1}{2 N !} N^{2} n_{\alpha}^{1} h_{\alpha \beta} n_{\beta}^{2} \psi_{1}^{*}\left(\mathbf{n}_{1}\right) \\
& \times \psi_{2}\left(\mathbf{n}_{2}\right)\left\langle 0\left|\left(n_{\gamma}^{1} \hat{\psi}_{\gamma}\right)^{N-1}\left(n_{\delta}^{2} \hat{\psi}_{\delta}^{\dagger}\right)^{N-1}\right| 0\right\rangle \\
&= \int d \mathbf{n} N n_{\alpha} h_{\alpha \beta} n_{\beta} \psi_{1}^{*}(\mathbf{n}) \psi_{2}(\mathbf{n}) .
\end{aligned}
$$

*Present address: Department of Physics and Astronomy, University of British Columbia, 6224 Agriculture Road, Vancouver, BC, Canada V6T 1 Z1.

${ }^{1}$ M. V. Berry, Proc. R. Soc. London, Ser. A 392, 45 (1984).

${ }^{2}$ B. Simon, Phys. Rev. Lett. 51, 2167 (1983).

${ }^{3}$ A. Tomita and R. Y. Chiao, Phys. Rev. Lett. 57, 937 (1986).

${ }^{4}$ D. Suter, G. C. Chingas, R. A. Harris, and A. Pines, Mol. Phys. 61, 1327 (1987).

${ }^{5}$ R. Tycko, Phys. Rev. Lett. 58, 2281 (1987).

${ }^{6}$ For a review on Berry's phases, see also A. Shapere and F. Wilczek, Geometric Phases (World Scientific, Singapore, 1989).

${ }^{7}$ F. D. M. Haldane, Phys. Rev. Lett. 50, 1153 (1983).

${ }^{8}$ F. D. M. Haldane, Phys. Rev. Lett. 61, 1029 (1988).

${ }^{9}$ N. Read and S. Sachdev, Phys. Rev. Lett. 62, 1694 (1989).

${ }^{10}$ F. Wilczek and A. Zee, Phys. Rev. Lett. 51, 2250 (1983).

${ }^{11}$ P. B. Wiegmann, Phys. Rev. Lett. 60, 821 (1988).

${ }^{12}$ X. G. Wen, F. Wilczek, and A. Zee, Phys. Rev. B 39, 11413 (1990).

${ }^{13}$ X. G. Wen, Phys. Rev. B 44, 2664 (1991).

${ }^{14}$ E. N. Bogachek and I. V. Krive, Phys. Rev. B 46, 14559 (1992).

${ }^{15}$ J. vonDelft and C. L. Henley, Phys. Rev. Lett. 69, 3236 (1992).

${ }^{16}$ D. Loss, D. P. DiVincenzo, and G. Grinstein, Phys. Rev. Lett. 69, 3232 (1992).

${ }^{17}$ N. V. Prokof'ev and P. E. Stamp, J. Phys.: Condens. Matter 5, L663 (1993).

${ }^{18}$ I. Tupitspyn, N. V. Prokofev, and P. C. E. Stamp, Int. J. Mod. Phys. B 11, 2901 (1997); I. Tupitspyn and B. Barbara, in Magnetoscience-From Molecules to Materials, edited by Miller and Dillon (Wiley, New York, 2001), Vol 3.

${ }^{19}$ B. Barbara, L. Thomas, F. Lionti, I. Chiorescu, and A. Sulpice, J.
Magn. Magn. Mater. 200, 167 (1999).

${ }^{20}$ W. Wernsdorfer and R. Sessoli, Science 284, 133 (2000).

${ }^{21}$ D. M. Stamper-Kurn, M. R. Andrews, A. P. Chikkatur, S. Inouye, H.-J. Miesner, J. Stenger, and W. Ketterle, Phys. Rev. Lett. 80, 2027 (1998).

${ }^{22}$ J. Stenger, S. Inouye, D. M. Stamper-Kurn, H.-J. Miesner, A. P. Chikkatur, and W. Ketterle, Nature (London) 396, 345 (1998).

${ }^{23}$ T. L. Ho, Phys. Rev. Lett. 81, 742 (1998).

${ }^{24}$ C. K. Law, H. Pu, and N. P. Bigelow, Phys. Rev. Lett. 81, 5257 (1998).

${ }^{25}$ T. Ohmi and K. Machinda, J. Phys. Soc. Jpn. 76, 1822 (1998).

${ }^{26}$ T. L. Ho and S. K. Yip, Phys. Rev. Lett. 84, 4031 (2000).

${ }^{27}$ F. Zhou, Phys. Rev. Lett. 87, 080401 (2001).

${ }^{28}$ F. Zhou, Int. J. Mod. Phys. B 17, 2643 (2003); cond-mat/ 0108473 (2001) (unpublished).

${ }^{29}$ E. Demler and F. Zhou, Phys. Rev. Lett. 88, 163001 (2002); E. Demler, F. Zhou, and D. F. M. Haldane, ITP-UU-01/09 (2001).

${ }^{30}$ Fei Zhou, Europhys. Lett. 63, 505 (2003); cond-mat/0207041 (unpublished).

${ }^{31}$ A. Imambekov, M. Lukin, and E. Demler, Phys. Rev. A 68, 063602 (2003).

${ }^{32}$ M. Snoek and F. Zhou, Phys. Rev. B 69, 094410 (2004).

${ }^{33}$ W. Vincent Liu and X.-G. Wen, cond-mat/0201187 (unpublished).

${ }^{34}$ F. Zhou and M. Snoek, Ann. Phys. (N.Y.) 308, 692 (2003).

${ }^{35}$ T. L. Ho and E. J. Mueller, Phys. Rev. Lett. 89, 050401 (2002).

${ }^{36}$ J. W. Reijnders, F. J. M. van Lankvelt, K. Schoutens, and N. Read, Phys. Rev. Lett. 89, 120401 (2002); Phys. Rev. A 69, 023612 (2004).

${ }^{37}$ L. D. Landau and E. M. Lifshitz, Quantum Mechanics (Pergamon, New York, 1958). 